\title{
EQUIVALENT FORMULATION AND NUMERICAL ANALYSIS OF A FIRE CONFINEMENT PROBLEM
}

\author{
Alberto Bressan ${ }^{1}$ AND TAO WANG $^{1}$
}

\begin{abstract}
We consider a class of variational problems for differential inclusions, related to the control of wild fires. The area burned by the fire at time $t>0$ is modelled as the reachable set for a differential inclusion $\dot{x} \in F(x)$, starting from an initial set $R_{0}$. To block the fire, a barrier can be constructed progressively in time. For each $t>0$, the portion of the wall constructed within time $t$ is described by a rectifiable set $\gamma(t) \subset \mathbb{R}^{2}$. In this paper we show that the search for blocking strategies and for optimal strategies can be reduced to a problem involving one single admissible rectifiable set $\Gamma \subset \mathbb{R}^{2}$, rather than the multifunction $t \mapsto \gamma(t) \subset \mathbb{R}^{2}$. Relying on this result, we then develop a numerical algorithm for the computation of optimal strategies, minimizing the total area burned by the fire.
\end{abstract}

Mathematics Subject Classification. 49Q20, 34A60, 49J24, 93B03.

Received December 12, 2008. Revised April 26, 2009.

Published online August 11, 2009.

\section{IntroduCtion}

This paper is concerned with the new class of problems introduced in [3], motivated by the control of forest fires or the spatial spreading of a contaminating agent. At each time $t \geq 0$, we denote by $R(t) \subset \mathbb{R}^{2}$ the region burned by the fire. In absence of control, the time evolution of the set $R(t)$ will be modelled in terms of a differential inclusion [2]. Let $F: \mathbb{R}^{2} \mapsto \mathbb{R}^{2}$ be a Lipschitz continuous multifunction with compact, convex values, and let $R_{0} \subset \mathbb{R}^{2}$ be a bounded, open set. At any given time $t \geq 0$, we then define $R(t)$ as the reachable set for the differential inclusion

$$
\dot{x} \in F(x) \quad x(0) \in R_{0},
$$

where the upper dot denotes a derivative w.r.t. time. In other words,

$$
R(t)=\left\{x(t) ; \quad x(\cdot) \text { absolutely continuous, } \quad x(0) \in R_{0}, \quad \dot{x}(\tau) \in F(x(\tau)) \text { for a.e. } \tau \in[0, t]\right\}
$$

We shall always assume that $0 \in F(x)$ for all $x \in \mathbb{R}^{2}$, which implies $R\left(t_{1}\right) \subseteq R\left(t_{2}\right)$ whenever $t_{1}<t_{2}$. We assume that the spreading of the fire can be controlled by constructing barriers. Here one may think of a thin strip of land which is either soaked with water poured from above (by airplane or helicopter), or sprayed with

\footnotetext{
Keywords and phrases. Dynamic blocking problem, differential inclusion, constrained minimum time problem.

1 Department of Mathematics, Penn State University University Park, Pa. 16802, USA. bressan@math.psu.edu; wang_t@math.psu.edu
} 
fire extinguisher, or cleared from all vegetation using a bulldozer. In any case, this will prevent the fire from crossing that particular strip of land.

In mathematical terms, we thus assume that the controller can construct a one-dimensional rectifiable curve $\gamma$ which blocks the spreading of the contamination. To model this, we consider a continuous, strictly positive function $\psi: \mathbb{R}^{2} \mapsto R_{+}$. Calling $\gamma(t) \subset \mathbb{R}^{2}$ the portion of the wall constructed within time $t \geq 0$, we make the following assumptions:

(H1) For any $t_{1}<t_{2}$ one has $\gamma\left(t_{1}\right) \subseteq \gamma\left(t_{2}\right)$.

(H2) For every $t \geq 0$, the wall satisfies

$$
\int_{\gamma(t)} \psi \mathrm{d} m_{1} \leq t
$$

where $m_{1}$ denotes the one-dimensional Hausdorff measure, normalized so that $m_{1}(\Gamma)$ yields the usual length of a smooth curve $\Gamma$.

In the above formula, $1 / \psi(x)$ is the speed at which the wall can be constructed, at the location $x$. In particular, if $\psi(x) \equiv \psi_{0}$ is constant, then (1.3) simply says that the length of the curve $\gamma(t)$ is $\leq t / \psi_{0}$. A strategy $\gamma$ satisfying (H1)-(H2) will be called an admissible strategy. In addition, we say that the strategy $\gamma$ is complete if it satisfies

(H3) For every $t \geq 0$ there holds

$$
\gamma(t)=\bigcap_{s>t} \gamma(s)
$$

Moreover, if $\gamma(t)$ has positive upper density at a point $x$, i.e. if

$$
\limsup _{r \rightarrow 0+} \frac{m_{1}(B(x, r) \cap \gamma(t))}{r}>0,
$$

then $x \in \gamma(t)$.

Here and in the sequel, $B(x, r)$ denotes the open disc centered at $x$ with radius $r$. As proved in [4], for every admissible strategy $t \mapsto \gamma(t)$ one can construct a second admissible strategy $t \mapsto \tilde{\gamma}(t) \supseteq \gamma(t)$, which is complete.

By constructing a barrier, the burned set can be reduced. Namely, we define

$$
\begin{aligned}
R^{\gamma}(t) \doteq\{x(t) ; & x(\cdot) \text { absolutely continuous, } x(0) \in R_{0}, \\
& \dot{x}(\tau) \in F(x(\tau)) \text { for a.e. } \tau \in[0, t], \quad x(\tau) \notin \gamma(\tau) \text { for all } \tau \in[0, t]\} .
\end{aligned}
$$

In the above setting, we consider the problem:

(BP1) Blocking Problem 1: Find an admissible strategy $t \mapsto \gamma(t)$ such that the corresponding reachable sets $R^{\gamma}(t)$ remain uniformly bounded, for all times $t \geq 0$.

In other words, calling $B_{r} \doteq B(0, r)=\left\{x \in \mathbb{R}^{2} ;|x|<r\right\}$, we seek a strategy such that

$$
R^{\gamma}(t) \subseteq B_{r} \quad \text { for all } t \geq 0
$$

for some radius $r$ sufficiently large.

To define an optimization problem, we need to introduce a cost functional. In general, this should take into account:

- the value of the area destroyed by the fire;

- the cost of building the barrier. 
We thus consider two continuous, non-negative functions $\alpha, \beta: \mathbb{R}^{2} \mapsto \mathbb{R}_{+}$and define the following functional

$$
J(\gamma)=\int_{R_{\infty}^{\gamma}} \alpha \mathrm{d} m_{2}+\int_{\gamma_{\infty}} \beta \mathrm{d} m_{1},
$$

where the sets $R_{\infty}^{\gamma}, \gamma_{\infty}$ are defined respectively as

$$
R_{\infty}^{\gamma} \doteq \bigcup_{t \geq 0} R^{\gamma}(t), \quad \gamma_{\infty} \doteq \bigcup_{t \geq 0} \gamma(t)
$$

The measurability of these sets was proved in [4]. In (1.6), $m_{2}$ denotes the two-dimensional Lebesgue measure, while $m_{1}$ is the one-dimensional Hausdorff measure. In the case of a fire, $\alpha(x)$ is the value of a unit area of land at the point $x$, while $\beta(x)$ is the cost of building a unit length of wall at the point $x$. This leads to:

(OP1) Optimization Problem 1: Find an admissible strategy $t \mapsto \gamma(t)$ for which the corresponding functional $J(\gamma)$ at (1.6) attains its minimum value.

For convenience, we list all the assumption below:

(A1) The initial set $R_{0}$ is open and bounded. Its boundary satisfies $m_{2}\left(\partial R_{0}\right)=0$.

(A2) The multifunction $F$ is Lipschitz continuous w.r.t. the Hausdorff distance. For each $x \in \mathbb{R}^{2}$ the set $F(x)$ is nonempty, closed and convex and contains the origin in its interior.

(A3) For every $x \in \mathbb{R}^{2}$ one has $\alpha(x) \geq 0, \beta(x) \geq \beta_{0}>0$, and $\psi(x) \geq \psi_{0}>0$. Moreover, $\alpha$ is locally integrable, while $\beta$ and $\psi$ are both lower semicontinuous.

As in [2], the Hausdorff distance between two compact sets $X, Y$ is defined as

$$
d_{H}(X, Y) \doteq \max \left\{\max _{x \in X} d(x, Y), \max _{y \in Y} d(y, X)\right\}
$$

where

$$
d(x, Y) \doteq \inf _{y \in Y} d(x, y)
$$

and $d(x, y) \doteq|x-y|$ is the Euclidean distance on $\mathbb{R}^{2}$. The multifunction $F$ is Lipschitz continuous if there exists a constant $L$ such that

$$
d_{H}(F(x), F(y)) \leq L \cdot d(x, y)
$$

for every couple of points $x, y$.

In its original formulation, a strategy is a mapping $t \mapsto \gamma(t) \subset \mathbb{R}^{2}$ describing the portion of the wall constructed at any given time $t \geq 0$. In the present paper we show that both the blocking problem and the optimal confinement problem can be reformulated in a simpler way, where a strategy is entirely determined by assigning one single rectifiable set $\Gamma \subset \mathbb{R}^{2}$. This provides a considerable simplification, useful for both analytical and numerical studies.

A first application of our equivalence result is given in the final section of this paper, developing a numerical algorithm which computes optimal strategies. Another application will appear in [5], providing a sharp estimate on the construction speed needed to block a fire on the half plane. We believe that the new formulation could also yield an alternative, more transparent proof of the existence of optimal strategies, studied in the earlier paper [4].

The paper is organized as follows. In Section 2 we provide the alternative formulations and state the main equivalence theorem. Sections 3 and 4 contain some geometric lemmas which play a key role in the proof. Roughly speaking, if two points $P, Q \in \mathbb{R}^{2}$ can be joined by some path $\gamma$ without crossing a rectifiable set $\Gamma$, then there is a second path $\gamma^{*}$ which still joins them without touching $\Gamma$, and has length $\leq|P-Q|+m_{1}(\Gamma)$. In Lemma 3.1 we prove a result of this type assuming that $\Gamma$ has finitely many compact connected components. The extension to the case where $\Gamma$ is any complete rectifiable set is then considered in Lemma 4.4. The proof refines some of the techniques introduced in [4]. Relying on Lemma 4.4, in Section 5 we can give a proof 
of the main equivalence theorem. Finally, in Section 6 we develop a numerical algorithm for the computation of optimal strategies. Numerical simulations are then compared with the explicit solutions obtained in $[3,6]$ by analytical methods.

\section{An EQUivalent FORMULATION}

Following [1,7], by a rectifiable set we mean a set $\Gamma \subset \mathbb{R}^{2}$ which can be decomposed as $\Gamma=\Gamma_{0} \cup \Gamma_{1}$, where $m_{1}\left(\Gamma_{0}\right)=0, m_{1}\left(\Gamma_{1}\right)<\infty$, and $\Gamma_{1}$ is contained in a countable union of Lipschitz continuous curves.

We say that the rectifiable set $\Gamma$ is complete if it contains all of its points of positive upper density:

$$
\theta^{*}(\Gamma ; x) \doteq \limsup _{r \rightarrow 0+} \frac{m_{1}(B(x, r) \cap \Gamma)}{r}>0 \quad \Longrightarrow \quad x \in \Gamma .
$$

We recall that, if $\Sigma$ is any rectifiable set (possibly not complete), the closure $\bar{\Sigma}$ may well be the entire plane $\mathbb{R}^{2}$. However, the union

$$
\Sigma^{*} \doteq \Sigma \cup\left\{x \in \mathbb{R}^{2} ; \limsup _{r \rightarrow 0+} \frac{m_{1}(B(x, r) \cap \Sigma)}{r}>0\right\}
$$

is a complete rectifiable set, with $m_{1}\left(\Sigma^{*}\right)=m_{1}(\Sigma)$. Throughout the following, we refer to $\Sigma^{*}$ as the completion of the set $\Sigma$.

Let now $\Gamma \subset \mathbb{R}^{2}$ be a complete rectifiable set. The reachable sets for the differential inclusion (1.1) restricted to $\mathbb{R}^{2} \backslash \Gamma$ are then defined as

$$
\begin{aligned}
R^{\Gamma}(t) \doteq\{x(t) ; & x(\cdot) \text { absolutely continuous, } \quad x(0) \in R_{0}, \quad \dot{x}(\tau) \in F(x(\tau)) \text { for a.e. } \tau \in[0, t], \\
& x(\tau) \notin \Gamma \text { for all } \tau \in[0, t]\} .
\end{aligned}
$$

As in (1.7), we also define $R_{\infty}^{\Gamma} \doteq \bigcup_{t \geq 0} R^{\Gamma}(t)$. Throughout the following, $\bar{S}$ will denote the closure of a set $S$. We say that the complete, rectifiable set $\Gamma$ is admissible in connection with the differential inclusion (1.1) and the bound on the construction speed (1.3) if, for every $t \geq 0$, the set

$$
\gamma(t) \doteq \Gamma \cap \overline{R^{\Gamma}(t)}
$$

satisfies (1.3), i.e. it can be constructed within time $t$. We now consider:

(BP2) Blocking Problem 2: Find an admissible rectifiable set $\Gamma \subset \mathbb{R}^{2}$ such that the corresponding reachable set $R_{\infty}^{\Gamma}$ is bounded.

(OP2) Optimization Problem 2: Find an admissible rectifiable set $\Gamma \subset \mathbb{R}^{2}$ such that the cost

$$
J(\Gamma)=\int_{R_{\infty}^{\Gamma}} \alpha \mathrm{d} m_{2}+\int_{\Gamma} \beta \mathrm{d} m_{1}
$$

attains the minimum possible value.

We will show that, under the assumptions (A1)-(A3), the two formulations of the blocking problem (BP1), (BP2) and of the optimization problem (OP1), (OP2) are essentially equivalent.

Toward this goal, given a rectifiable set $\Gamma$, we define the corresponding multifunction $t \mapsto \gamma(t)$ according to (2.2). Vice versa, given an admissible strategy $\gamma(\cdot)$, we consider the set

$$
\Gamma \doteq \text { completion of }\left(\gamma_{\infty} \backslash R_{\infty}^{\gamma}\right)
$$

where $\gamma_{\infty}, R_{\infty}^{\gamma}$ are the sets defined at (1.7). Our main results are as follows. 
Theorem 2.1. Let the assumptions (A1)-(A3) hold. Then there exists an admissible strategy $t \mapsto \gamma(t)$ with $m_{1}\left(\gamma_{\infty}\right)<\infty$ which solves the blocking problem (BP1) if and only if there exists an admissible rectifiable set $\Gamma$ which solves (BP2).

Theorem 2.2. Let the assumptions (A1)-(A3) hold.

(i) If $t \mapsto \gamma(t)$ is a complete, optimal strategy for the minimization problem (OP1), then the set $\Gamma$ defined at (2.4) is admissible and provides an optimal solution to the minimization problem (OP2).

(ii) Vice versa, if $\Gamma$ is a complete, rectifiable set which is admissible, and optimal for the problem (OP2), then the strategy $\gamma$ defined at (2.2) is admissible and provides an optimal solution to the minimization problem (OP1).

Remark. According to our earlier definition, a rectifiable set $\Gamma$ must have finite length. This motivates the requirement $m_{1}\left(\gamma_{\infty}\right)<\infty$ in Theorem 2.1. According to Lemma 4.1 in the forthcoming paper [5], this additional requirement can be dropped at least in the isotropic case, where $F(x) \equiv \bar{B}(0, r)$ for all $x \in \mathbb{R}^{2}$. We also observe that, since in (A3) we put a strictly positive $\operatorname{cost} \beta(x)>\beta_{0}>0$ on the length of the wall, any optimal strategy must generate a wall of finite length.

By the analysis in [4], Theorem 2.2 yields the following regularity result.

Corollary 2.1. Under the assumptions (A1)-(A3), if there exists an admissible set $\Gamma$ such that $J(\Gamma)<\infty$, then the optimal confinement problem (OP2) has a solution $\Gamma^{*}$ of the form

$$
\Gamma^{*}=\left(\bigcup_{i \geq 1} \Gamma_{i}\right) \cup \mathcal{N}
$$

where each $\Gamma_{i}$ is a compact connected rectifiable set, and $m_{1}(\mathcal{N})=0$.

\section{Going AROUnd WALlS}

Let $\Gamma$ be a rectifiable set, and assume that the two points $Q_{0}, Q_{1}$ lie in the same connected component of the complement $\mathbb{R}^{2} \backslash \Gamma$. Then we expect that they can be connected by a path of length arbitrarily close to $\left|Q_{0}-Q_{1}\right|+m_{1}(\Gamma)$. The next lemma shows that this is true, assuming that $\Gamma$ has finitely many connected components. A more general result will be proved in a subsequent section.

Lemma 3.1. Let $\Gamma=\Gamma_{1} \cup \ldots \cup \Gamma_{N} \subset \mathbb{R}^{2}$ be the union of finitely many compact, connected rectifiable sets. Assume that the points $Q_{0}, Q_{1} \notin \Gamma$ can be connected by a continuous path $\gamma:[0,1] \mapsto \mathbb{R}^{2}$ which does not intersect $\Gamma$. Then, for every $\varepsilon>0$ there exists a Lipschitz continuous path $\gamma^{*}:[0,1] \mapsto \mathbb{R}^{2} \backslash \Gamma$ with $\gamma^{*}(0)=Q_{0}$, $\gamma^{*}(1)=Q_{1}$ whose total length satisfies

$$
\left\|\gamma^{*}\right\| \doteq \int_{0}^{1}\left|\dot{\gamma}^{*}(s)\right| \mathrm{d} s<\left|Q_{1}-Q_{0}\right|+m_{1}(\Gamma)+\varepsilon .
$$

Proof. 1. As a preliminary, we recall some basic facts from geometric measure theory. Let $K \subset \mathbb{R}^{2}$ be a compact, connected, rectifiable set with finite length. For each $r>0$ define the compact neighborhood

$$
K_{r} \doteq\left\{x \in \mathbb{R}^{2} ; d(x, \Gamma) \leq r\right\} .
$$

Then

$$
\lim _{r \rightarrow 0+} \frac{m_{2}\left(K_{r}\right)}{2 r}=m_{1}(K) .
$$

Indeed, this follows from Theorem 3.2.39 in [8], p. 275. An application of the co-area formula [1] shows that the boundaries of the sets $K_{r}$ satisfy

$$
\liminf _{r \rightarrow 0+} m_{1}\left(\partial K_{r}\right) \leq 2 m_{1}(K)
$$


2. By an approximation argument, we can assume that the curve $\gamma$ joining $Q_{0}$ with $Q_{1}$ is a polygonal, having finitely many intersections with the segment (see Fig. 1)

$$
S \doteq\left\{\theta Q_{1}+(1-\theta) Q_{0} ; \quad \theta \in[0,1]\right\}
$$

joining $Q_{0}$ with $Q_{1}$. More precisely, let $P_{i}=\gamma\left(t_{i}\right) \in S$, be these intersection points, with $0=t_{0}<t_{1}<\ldots<$ $t_{N}=1$. By transversality, for every given $r>0$ sufficiently small, one can determine parameter values

$$
0=t_{0}<t_{0}^{+}<t_{1}^{-}<t_{1}<t_{1}^{+}<\ldots<t_{i}^{-}<t_{i}<t_{i}^{+}<\ldots<t_{N}^{-}<t_{N}=1
$$

such that

$$
\begin{cases}d(\gamma(t), S)>r & \text { for } t_{i-1}^{+}<t<t_{i}^{-} \\ d(\gamma(t), S)=r & \text { for } t=t_{i}^{-} \text {and } t=t_{i}^{+} \\ d(\gamma(t), S)<r & \text { for } t_{i}^{-}<t<t_{i}^{+} \\ d(\gamma(t), \Gamma)>r & \text { for all } t \in[0,1] .\end{cases}
$$

Consider the compact connected, rectifiable set

$$
K \doteq S \cup\left(\bigcup_{1 \leq i \leq N, \Gamma_{i} \cap S \neq \emptyset} \Gamma_{i}\right)
$$

together with its compact, connected neighborhood

$$
K_{r} \doteq\{x ; \quad d(x, K) \leq r\}
$$

Choose a radius $\bar{r}$ with $0<\bar{r}<1$, such that

$$
\Gamma_{j} \cap K_{\bar{r}}=\emptyset \quad \text { whenever } \quad \Gamma_{j} \cap S=\emptyset,
$$

and also

$$
m_{1}\left(\Pi_{\bar{r}}\right)<\varepsilon
$$

Here for any $r>0$ suitably small we define

$$
\Pi_{r} \doteq\left\{\gamma(t) ; \quad t \in\left[0, t_{0}^{+}\right] \cup\left[t_{1}^{-}, t_{1}^{+}\right] \cup \ldots \cup\left[t_{N-1}^{-}, t_{N-1}^{+}\right] \cup\left[t_{N}^{-}, 1\right]\right\}=\gamma([0,1]) \cap K_{r} .
$$

3. By (3.2) there exists a radius $0<r_{2}<\bar{r}$ such that

$$
m_{2}\left(K_{r_{2}}\right)<2 r_{2}\left(m_{1}(K)+\varepsilon\right) .
$$

Choosing $r_{1}, \varepsilon^{\prime}$ sufficiently small, with $0<\varepsilon \ll<r_{1}<<r_{2}$, we achieve

$$
m_{2}\left(K_{r_{2}} \backslash K_{r_{1}}\right)<2\left(r_{2}-r_{1}\right)\left(m_{1}(K)+2 \varepsilon\right) .
$$

Define the distance function with cutoff

$$
V(x) \doteq\left\{\begin{array}{rll}
d(x, K) & \text { if } \quad r_{1}<d(x, K)<r_{2}, \\
r_{1} & \text { if } d(x, K) \leq r_{1}, \\
r_{2} & \text { if } d(x, K) \geq r_{2} .
\end{array}\right.
$$


Let $V_{\varepsilon^{\prime}} \doteq \varphi_{\varepsilon^{\prime}} * V$ be a mollification of $V$, where the smooth kernel $\varphi_{\varepsilon^{\prime}}$ is supported inside the disc $B\left(0, \varepsilon^{\prime}\right)$. The functions $V, V_{\varepsilon^{\prime}}$ are both Lipschitz continuous with constant one. Using the co-area formula and then (3.6), we obtain

$$
\begin{aligned}
\int_{r_{1}}^{r_{2}} m_{1}\left(\left\{x ; V_{\varepsilon^{\prime}}(x)=s\right\}\right) \mathrm{d} s & =\int_{\mathbb{R}^{2}}\left|\nabla V_{\varepsilon^{\prime}}\right| \mathrm{d} x \leq \int_{\mathbb{R}^{2}}|\nabla V| \mathrm{d} x \\
& =m_{2}\left(K_{r_{2}} \backslash K_{r_{1}}\right)<2\left(r_{2}-r_{1}\right)\left(m_{1}(K)+2 \varepsilon\right) .
\end{aligned}
$$

Since $V_{\varepsilon^{\prime}}$ is smooth, by Sard's theorem almost every level set $\Sigma_{r} \doteq\left\{x ; V_{\varepsilon^{\prime}}(x)=r\right\}$ is the finite union of finitely many smooth curves. By (3.8), there exists some $\rho$, with $r_{1}<\rho<r_{2}$ such that

$$
m_{1}\left(\Sigma_{\rho}\right)<2 m_{1}(K)+4 \varepsilon
$$

The construction of $V_{\varepsilon^{\prime}}$ clearly implies

$$
\Sigma_{\rho} \subset K_{r_{2}+\varepsilon^{\prime}} \backslash K_{r_{1}-\varepsilon^{\prime}} .
$$

Consider the sub-level set

$$
\Sigma_{\rho}^{-} \doteq\left\{x ; V_{\varepsilon^{\prime}}(x)<\rho\right\}
$$

Observe that this open set need not be connected. However, since $\Sigma_{\rho}^{-} \supset K_{r_{1}-\varepsilon^{\prime}}$ and $K_{r_{1}-\varepsilon^{\prime}}$ is connected, we can uniquely define the set $\widetilde{\Sigma}_{\rho}$ as the connected component of $\Sigma_{\rho}^{-}$which contains $K_{r_{1}-\varepsilon^{\prime}}$. Clearly, its boundary satisfies

$$
\partial \widetilde{\Sigma}_{\rho} \subseteq \partial \Sigma_{\rho}^{-} \subseteq \Sigma_{\rho}
$$

4. Call $\Omega_{1}, \ldots, \Omega_{\nu}$ the connected components of $\mathbb{R}^{2} \backslash \widetilde{\Sigma}_{\rho}$. We observe that, since $\widetilde{\Sigma}_{\rho}$ is connected, every bounded component $\Omega_{j}$ is simply connected (see for instance Thm. 4 in Chap. X of [10], p. 512, or similar results in [9,11]). Moreover, the boundary $\partial \Omega_{j}$ of every component of $\mathbb{R}^{2} \backslash \widetilde{\Sigma}$ (including the unbounded connected component) is a smooth Jordan curve.

As the parameter $s$ ranges in $[0,1]$, the point $\gamma(s)$ on the polygonal path may move in and out of a given component $\Omega_{i}$ several times. To cope with this situation, we define the times

$$
\tau_{i}^{-} \doteq \inf \left\{t \in[0,1] ; \gamma(t) \in \Omega_{i}\right\}, \quad \tau_{i}^{+} \doteq \sup \left\{t \in[0,1] ; \gamma(t) \in \Omega_{i}\right\}
$$

We remark that, since the components $\Omega_{i}$ are strictly bounded away from each other, the times $\tau_{1}^{ \pm}, \ldots, \tau_{\nu}^{ \pm}$are all distinct.

We now define a sequence of indices $j_{1}, \ldots, j_{M} \in\{1, \ldots, \nu\}$ inductively as follows.

- We begin by setting $j_{1}$ be the unique index such that

$$
\tau_{j_{1}}^{-}=\min \left\{\tau_{1}^{-}, \ldots, \tau_{\nu}^{-}\right\}
$$

- By induction, assume that $j_{1}, \ldots, j_{k}$ have been defined. Two cases can occur.

- If $\gamma(s) \in \widetilde{\Sigma}_{\rho}$ for all $s \in\left[\tau_{j_{k}}, 1\right]$, then we set $M=k$ and the construction terminates. 


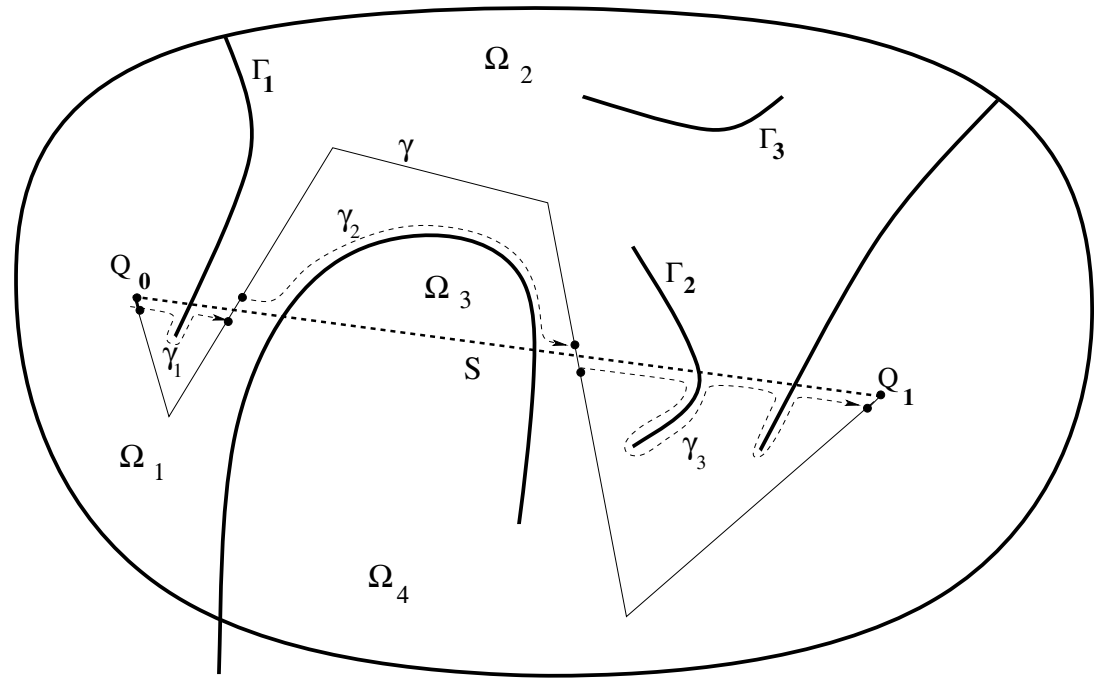

Figure 1. Construction of the path $\gamma^{*}$. Here $K=S \cup \Gamma_{1} \cup \Gamma_{2}, M=3, j_{1}=1, j_{2}=2, j_{3}=4$.

- Otherwise, we let $j_{k+1}$ be the unique index such that

$$
\tau_{j_{k+1}}^{-}=\min \left\{\tau_{i}^{-} ; 1 \leq i \leq \nu, \tau_{i}^{-}>\tau_{j_{k}}^{+}\right\}
$$

and continue the induction procedure.

By the above construction, it is clear that the indices $j_{1}, \ldots, j_{M}$ are all distinct, and that the inductive procedure must terminate after a number of steps $\leq \nu$.

5. Consider the points

$$
P_{i}^{-}=\gamma\left(\tau_{j_{i}}^{-}\right), \quad P_{i}^{+}=\gamma\left(\tau_{j_{i}}^{+}\right), \quad i=1, \ldots, M
$$

along the boundary of $\Omega_{j_{i}}$. Since this boundary is a smooth closed curve, we can start from $P_{i}^{-}$and move along $\partial \Omega_{j_{i}}$ in two opposite directions. In both cases, we eventually reach $P_{j_{i}}^{+}$. In other words, there exists two paths joining $P_{i}^{-}$with $P_{i}^{+}$, both contained inside $\partial \Omega_{j_{i}}$. The sum of their lengths is precisely $m_{1}\left(\partial \Omega_{j_{i}}\right)$. Choosing the shorter one, we obtain a path $\gamma_{i}:[0,1] \mapsto \partial \Omega_{j_{i}}$ with

$$
\gamma_{i}(0)=P_{i}^{-}, \quad \gamma_{i}(1)=P_{i}^{+}, \quad\left\|\gamma_{i}\right\| \doteq \int_{0}^{1}\left|\dot{\gamma}_{i}(s)\right| \mathrm{d} s \leq \frac{1}{2} m_{1}\left(\partial \Omega_{j_{i}}\right) .
$$

6. Concerning the remaining portion of the path $\gamma$, we have

$$
\widetilde{\Gamma} \doteq\left\{\gamma(t) ; t \in\left[0, \tau_{j_{1}}^{-}\right] \cup\left[\tau_{j_{1}}^{+}, \tau_{j_{2}}^{-}\right] \cup \ldots \cup\left[\tau_{j_{\nu}}^{+}, 1\right]\right\} \subset K_{r_{2}+\varepsilon^{\prime}} \subset K_{\bar{r}} .
$$

Recalling (3.5) we thus have

$$
m_{1}(\widetilde{\Gamma}) \leq m_{1}\left(\Pi_{\bar{r}}\right)<\varepsilon
$$

7. The path $\gamma^{*}$ connecting $Q_{0}$ with $Q_{1}$ is now obtained as a concatenation of the following pieces (see Fig. 1):

- the paths $\gamma_{i}$ joining $P_{i}^{-}$with $P_{i}^{+}$, for $i=1, \ldots, M$;

- the segments $S_{i}$ joining $P_{i}^{+}$with $P_{i+1}^{-}$, for $i=1, \ldots, M-1$; 
- the segment $S_{0}$ joining $Q_{0}$ with $P_{1}^{-}$;

- the segment $S_{M}$ joining $P_{M}^{+}$with $Q_{1}$.

After a suitable reparametrization, we thus obtain a Lipschitz continuous path $\gamma^{*}:[0,1] \mapsto \mathbb{R}^{2} \backslash \Gamma$ with $\gamma^{*}(0)=Q_{0}, \gamma^{*}(1)=Q_{1}$, whose total length satisfies

$$
\begin{aligned}
\left\|\gamma^{*}\right\| & \doteq \int_{0}^{1}\left|\dot{\gamma}^{*}(s)\right| \mathrm{d} s \leq \sum_{i=1}^{M} \frac{1}{2} m_{1}\left(\partial \Omega_{j_{i}}\right)+m_{1}(\widetilde{\Gamma}) \\
& \leq \frac{1}{2} m_{1}\left(\Sigma_{\rho}\right)+m_{1}\left(\Pi_{\bar{r}}\right)<\frac{1}{2}\left(2 m_{1}(K)+4 \varepsilon\right)+\varepsilon=\left|Q_{1}-Q_{0}\right|+m_{1}(\Gamma)+3 \varepsilon,
\end{aligned}
$$

because of (3.9) and (3.5). Since $\varepsilon>0$ was arbitrary, this proves (3.1).

\section{Additional lemmas}

In this section we establish some intermediate results, which will be used in the proof of the main theorem. By a continuum we mean a compact connected set. Given a set $\Gamma \subset \mathbb{R}^{2}$ and a vector $v \in \mathbb{R}^{2}$, we consider the shifted set

$$
\mathcal{T}^{v} \Gamma \doteq\{x+v ; \quad x \in \Gamma\} .
$$

For convenience, we recall here the result proved in Lemma 2.9 of [4].

Lemma 4.1. Let $Q=[0,1] \times[0,1]$ be the unit square in $\mathbb{R}^{2}$. Let $J_{0}$ and $J_{1}$ be any two sides. For any $\varepsilon>0$ there exists $\eta>0$ with the following property. If $W \subset Q$ is a set with $m_{1}(W) \leq \eta$, then there is a set $K_{0} \subseteq J_{0}$ such that

(i) $m_{1}\left(K_{0}\right)>1-\varepsilon$;

(ii) for every $x \in K_{0}$, the set $I_{x}$ of points $y \in J_{1}$ for which the segment $[x, y]$ with endpoints $x, y$ does not intersect $W$ has measure $m_{1}\left(I_{x}\right)>1-\varepsilon$.

The next lemma shows that a polygonal curve can be shifted by a small amount, so that its intersection with a rectifiable set becomes arbitrarily small.

Lemma 4.2. Let $\Sigma$ be a polygonal, and let $\Gamma_{0} \subset \mathbb{R}^{2}$ be a rectifiable set, with $m_{1}\left(\Gamma_{0}\right)<\infty$. Then, for every $\rho, \varepsilon>0$, there exists a vector $v \in \mathbb{R}^{2}$ such that

$$
|v| \leq \rho, \quad m_{1}\left(\mathcal{T}^{v} \Sigma \cap \Gamma_{0}\right) \leq \varepsilon .
$$

Proof. Let $\Sigma=\Sigma_{1} \cup \ldots \cup \Sigma_{N}$, where each $\Sigma_{i}$ is an edge of the polygonal. Choose a unit vector $\mathbf{e} \in \mathbb{R}^{2}$ which is not parallel to any of the edges $\Sigma_{i}$. Fix an integer $M>\varepsilon^{-1} N m_{1}\left(\Gamma_{0}\right)$ and define the vectors

$$
v_{j} \doteq \frac{j \rho}{M} \mathbf{e}, \quad j=1, \ldots, M .
$$

Moreover, consider the function

$$
\phi(j) \doteq m_{1}\left(\mathcal{T}^{v_{j}} \Sigma \cap \Gamma_{0}\right)=\sum_{i=1}^{N} m_{1}\left(\mathcal{T}^{v_{j}} \Sigma_{i} \cap \Gamma_{0}\right) .
$$

For each $i$, the shifted sets $\mathcal{T}^{v_{0}} \Sigma_{i}, \ldots, \mathcal{T}^{v_{M}} \Sigma_{i}$ are all disjoint. Therefore

$$
\sum_{j=1}^{M} \phi(j) \leq \sum_{i=1}^{N} \sum_{j=1}^{M} m_{1}\left(\mathcal{T}^{v_{j}} \Sigma_{i} \cap \Gamma_{0}\right) \leq N m_{1}\left(\Gamma_{0}\right) .
$$


Hence there exists some index $j^{*} \in\{1, \ldots, M\}$ such that

$$
\phi\left(j^{*}\right) \leq \frac{N m_{1}\left(\Gamma_{0}\right)}{M}<\varepsilon
$$

Setting $v \doteq v_{j^{*}}$ we achieve the desired conclusion.

The next lemma, which also follows from the analysis in [4], shows that if a point $q$ does not belong to a complete rectifiable set $\Gamma$, then most of the points near $q$ can be reached from $q$ without crossing $\Gamma$.

Lemma 4.3. Let $\Gamma \subset \mathbb{R}^{2}$ be a complete rectifiable set, and assume $q \notin \Gamma$. Then, for every $\varepsilon>0$, there exists $\left.r_{0} \in\right] 0, \varepsilon\left[\right.$ such that the following holds. Every point on the circumference $\partial B\left(q, r_{0}\right)=\left\{x \in \mathbb{R}^{2} ; \quad|x-q|=r_{0}\right\}$ can be connected to $q$ by a path of length $\leq(1+4 \pi) r_{0}$ which does not intersect $\Gamma$.

Proof. By the assumptions,

$$
\lim _{r \rightarrow 0} \frac{m_{1}(B(q, r) \cap \Gamma)}{r}=0
$$

Hence there exists $0<\rho<\varepsilon$ such that

$$
m_{1}(B(q, r) \cap \Gamma)<\frac{r}{5} \quad \text { for all } r<\rho .
$$

We claim that, for each integer $j \geq 0$ there exists at least one radius $r_{j} \in\left[2^{-j-1} \rho, 2^{-j} \rho\right]$ such that the circumference $\partial B\left(q, r_{j}\right)$ does not intersect $\Gamma$. Otherwise, $m_{1}\left(B\left(q, 2^{-j} \rho\right) \cap \Gamma\right) \geq 2^{-j-1} \rho$, providing a contradiction with (4.2). In addition, for each $j \geq 0$, there must exist a unit vector $v_{j} \in \mathbb{R}^{2}$ such that the segment

$$
S_{j} \doteq\left\{\lambda v_{j} ; \lambda \in\left[2^{-j-2} \rho, 2^{-j} \rho\right]\right\}
$$

does not intersect $\Gamma$. Otherwise, $m_{1}\left(B\left(q, 2^{-j} \rho\right) \cap \Gamma\right) \geq 2 \pi \cdot 2^{-j-2} \rho$, providing again a contradiction.

As shown in Figure 2, for each integer $j \geq 0$ consider the point $b_{j}$ at the intersection of the circumference $\partial B\left(q, r_{j}\right)$ and the segment $S_{j}$. For $j \geq 1$, let $a_{j}$ be the point at the intersection of $S_{j-1}$ and $\partial B\left(q, r_{j}\right)$.

Given any point $x$ on the circumference $\partial B\left(q, r_{0}\right)$, a path connecting $x$ with $q$ is now constructed as follows. Move from $x$ to $b_{0}$ along the shorter of the two arcs on the circumference $\partial B\left(q, r_{0}\right)$, then from $b_{0}$ to $a_{1}$ along the segment $S_{0}$. By induction, after the point $a_{j}$ is reached, we move from $a_{j}$ to $b_{j}$ along the shorter arc of circumference $\partial B\left(q, r_{j}\right)$, then from $b_{j}$ to $a_{j+1}$ along the segment $S_{j+1}$. Continuing in this way, we obtain a continuous curve $\gamma$ joining $x$ with $q$. Its total length is computed by

$$
m_{1}(\gamma)=\sum_{j \geq 1} m_{1}\left(\gamma \cap S_{j}\right)+\sum_{j \geq 0} m_{1}\left(\gamma \cap B\left(q, r_{j}\right)\right) \leq r_{0}+\sum_{j \geq 0} 2^{-j} \pi \rho \leq r_{0}+4 \pi r_{0},
$$

as claimed.

The main result of this section extends Lemma 3.1 to general rectifiable sets. As a preliminary, we observe that every complete rectifiable set $\Gamma$ can be decomposed as

$$
\Gamma=\bigcup_{k \geq 0} \Gamma_{k}
$$

where $\Gamma_{0}$ is totally disconnected while, for $k \geq 1$, the sets $\Gamma_{k}$ are disjoint compact connected components with strictly positive length.

Lemma 4.4. Let $\Gamma \subset \mathbb{R}^{2}$ be a complete rectifiable set, decomposed as in (4.3). Let $q_{0}, q_{1} \notin \Gamma$ be any two points contained in the same connected component of $\mathbb{R}^{2} \backslash \Gamma$. Then, for every $\varepsilon>0$, there exists a Lipschitz continuous 
path $Y^{\sharp}:[0,1] \mapsto \mathbb{R}^{2} \backslash \Gamma$ with $Y^{\sharp}(0)=q_{0}, Y^{\sharp}(1)=q_{1}$, and whose total length satisfies

$$
\left\|Y^{\sharp}\right\| \leq\left|q_{1}-q_{0}\right|+\sum_{k \geq 1} m_{1}\left(\Gamma_{k}\right)+\varepsilon \doteq L .
$$

Proof. We shall follow the argument in Section 8 of [4], based on the construction of a grid of black and white squares, and of a polygonal trajectory living on white squares, which avoids the set $\Gamma$. We divide the argument in several steps.

1. Let $0<\varepsilon<<1$ be given. By Lemma 4.3, there exists a radius $0<r_{0}<\varepsilon$ such that every point on the circumference $\partial B\left(q_{0}, r_{0}\right)$ can be connected to $q_{0}$ by a path of length $<(1+4 \pi) r_{0}$ without crossing $\Gamma$. Similarly, there exists $0<r_{1}<\varepsilon$ such that every point on the circumference $\partial B\left(q_{1}, r_{1}\right)$ can be connected to $q_{1}$ by a path of length $<(1+4 \pi) r_{1}$ without crossing $\Gamma$.

2. Let $0<\eta<1$ be a constant for which the conclusions of Lemma 4.1 hold. Then choose a constant $\delta>0$ such that

$$
\delta<\frac{\varepsilon \eta}{100}, \quad \delta<\frac{\min \left\{r_{0}, r_{1}\right\}}{100} .
$$

Finally, recalling the decomposition (4.3), choose an integer $N \geq 1$ such that

$$
\sum_{k>N} m_{1}\left(\Gamma_{k}\right)<\delta
$$

3. From the assumptions it follows that the points $q_{0}, q_{1}$ lie in the same connected component of $V_{N} \doteq$ $\mathbb{R}^{2} \backslash\left(\bigcup_{k=1}^{N} \Gamma_{k}\right)$. Since each $\Gamma_{k}$ is compact, the set $V_{N}$ is open. Hence its connected components are pathconnected, and there exists a continuous path $\gamma:[0,1] \mapsto V_{N}$ connecting $q_{0}$ with $q_{1}$.

Applying Lemma 3.1, with $\Gamma$ replaced by the finite union $\Gamma_{1} \cup \ldots \cup \Gamma_{N}$, we obtain a continuous, piecewise affine map

$$
Y:[0,1] \mapsto \mathbb{R}^{2} \backslash\left(\bigcup_{1 \leq k \leq N} \Gamma_{k}\right)
$$

with $Y(0)=q_{0}, Y(1)=q_{1}$, and having length $\|Y\| \leq L$, where $L$ is the constant defined at (4.4). For convenience, we define the set $\Sigma \doteq\{Y(s) ; s \in[0,1]\} \subset \mathbb{R}^{2}$. Observe that, since $\Sigma \cap \Gamma_{k}=\emptyset$ for all $k=1, \ldots, N$, by compactness we have

$$
\delta_{N} \doteq \min \left\{|x-y| ; \quad x \in \Sigma, \quad y \in \bigcup_{1 \leq k \leq N} \Gamma_{k}\right\}>0 .
$$

4. Using Lemma 4.2, we can choose a vector $v \in \mathbb{R}^{2}$ such that $|v|<\min \left\{\delta, \delta_{N}\right\}$ and moreover the shifted set $\Sigma^{v} \doteq \mathcal{T}^{v} \Sigma=\{v+x ; \quad x \in \Sigma\}$ satisfies

$$
m_{1}\left(\Gamma_{0} \cap \Sigma^{v}\right)<\delta .
$$

Notice that this choice of $v$ implies

$$
\Sigma^{v} \cap \Gamma_{k}=\emptyset \quad 1 \leq k \leq N .
$$

Calling $B\left(\Sigma^{v}, r\right)$ the open neighborhood of radius $r$ around the set $\Sigma^{v}$, by (4.8) there exists $\rho_{1}>0$ small enough so that

$$
m_{1}\left(\Gamma_{0} \cap B\left(\Sigma^{v}, 2 \rho_{1}\right)\right)<\delta, \quad B\left(\Sigma^{v}, 2 \rho_{1}\right) \cap \Gamma_{k}=\emptyset \quad 1 \leq k \leq N .
$$


Since the total length of $\Gamma$ is finite, there can be at most finitely many connected components $\Gamma_{i(1)}, \ldots, \Gamma_{i(m)}$ which intersect $\Sigma^{v}$ and also contain a point $x \notin B\left(\Sigma^{v}, \rho_{1}\right)$. Since $\Gamma_{0}$ is disjoint from all $\Gamma_{k}$, we can now choose $\rho_{2}>0$ sufficiently small, so that

$$
m_{1}\left(\Gamma_{0} \cap \bigcup_{\ell=1}^{m} B\left(\Gamma_{i(\ell)}, \rho_{2}\right)\right)<\delta, \quad B\left(\Gamma_{i(\ell)}, \rho_{2}\right) \cap \Gamma_{k}=\emptyset \quad 1 \leq \ell \leq m, \quad 1 \leq k \leq N .
$$

5. In the following we denote by $s \mapsto Y^{v}(s) \doteq v+Y(s)$ the shifted map. By (4.6), (4.8), and (4.9), the total length of all connected components of $\Gamma$ which intersect the shifted path $\Sigma^{v} \doteq\left\{Y^{v}(s) ; s \in[0,1]\right\}$ is $<2 \delta$. To get around these small components, a further construction is needed.

Choose coordinates $\left(w_{1}, w_{2}\right)$ on $\mathbb{R}^{2}$. By a suitable rotation, we can assume that every vertical and horizontal line intersects $\Gamma$ in a set of zero length.

Given a mesh size $\sigma>0$, whose precise value will be specified later, we consider the grid formed by the lines $\left\{w_{i}=j \sigma\right\}$ for $i=1,2$ and $j \in \mathbb{Z}$. Points of the form $\left(w_{1}, w_{2}\right)=\left(j_{1} \sigma, j_{2} \sigma\right)$ with $j_{1}, j_{2} \in \mathbb{Z}$ are the vertices of the grid, while horizontal or vertical segments of length $\sigma$ connecting two vertices will be called edges of the grid. Finally, the sets of type $\left[j_{1} \sigma,\left(j_{1}+k\right) \sigma\right] \times\left[j_{2} \sigma,\left(j_{2}+k\right) \sigma\right]$ will be called squares of size $k \sigma$ of the grid. When not specified, we understand that the size of the square is $\sigma$. By a translation of coordinates, it is not restrictive to assume that the path $Y^{v}$ does not touch any of the vertices of the grid.

6. We now choose times $\tau_{0}<\tau_{1}<\ldots<\tau_{M} \leq 1$ and corresponding squares $Q_{i}$ of the grid, containing the points $Y^{v}\left(\tau_{i}\right)$, with the following inductive procedure.

We begin by setting $\tau_{0}=0$ and choosing a square $Q_{0}$ such that $Y^{v}\left(\tau_{0}\right)=q_{0}+v \in Q_{0}$.

Now assume that $\tau_{i}$ has been chosen, together with a closed square $Q_{i}$ of the grid containing $Y^{v}\left(\tau_{i}\right)$. Consider the eight squares surrounding $Q_{i}$ which, together with $Q_{i}$, form a square $\widetilde{Q}_{i}$ of size $3 \sigma$. Two cases are considered:

- If in the interval $\left[\tau_{i}, 1\right]$ the trajectory $Y^{v}$ remains in $\widetilde{Q}_{i}$, then we set $i=M$ and the inductive procedure terminates.

- Otherwise, we let $\tau_{i+1}$ be the first time $t>\tau_{i}$ such that $Y(t) \in \partial \widetilde{Q}_{i}$.

Note that, at the time $\tau_{M}$ when the inductive procedure stops, we have $\left|Y^{v}\left(\tau_{M}\right)-Y^{v}(1)\right| \leq 2 \sqrt{2} \sigma$. Moreover, the above construction yields

$$
\sigma \leq\left|Y^{v}\left(\tau_{i+1}\right)-Y^{v}\left(\tau_{i}\right)\right| \leq 2 \sqrt{2} \sigma \quad i=0,1, \ldots, M-1 .
$$

The path $Y^{v}$ can be interpolated by a piecewise affine map $Y^{\sigma}:\left[0, \tau_{M}\right] \rightarrow \mathbb{R}^{2}$ by setting (see Fig. 3)

$$
Y^{\sigma}(t)=\frac{t-\tau_{i}}{\tau_{i+1}-\tau_{i}} Y^{v}\left(\tau_{i}\right)+\frac{\tau_{i+1}-t}{\tau_{i+1}-\tau_{i}} Y^{v}\left(\tau_{i+1}\right) \quad \text { for } t \in\left[\tau_{i}, \tau_{i+1}\right] .
$$

For notational convenience, we call $\xi_{0}, \xi_{1}, \ldots, \xi_{\bar{M}}$ the points where the polygonal $Y^{\sigma}$ intersects the edges of the grid. Notice that, in general, we have $\bar{M} \geq M$. However, our construction yields a lower bound on the distances between three consecutive points, namely

$$
\left|\xi_{i+2}-\xi_{i+1}\right|+\left|\xi_{i+1}-\xi_{i}\right|+\left|\xi_{i}-\xi_{i-1}\right| \geq \sigma
$$

for every $i \in\{1, \ldots, \bar{M}-2\}$.

7. Each square $Q$ of the grid will be classified as black or white, depending on the relative size of the intersection $Q \cap \Gamma$. More precisely, all the closed squares $Q$ of the grid for which

$$
m_{1}(\Gamma \cap Q) \geq \eta \sigma
$$


will be called black. The other closed squares will be called white. It is useful here to recall that, by the choice of the coordinates $\left(w_{1}, w_{2}\right)$, we always have $m_{1}(\Gamma \cap \partial Q)=0$.

As in [4], by a path of squares we mean an ordered collection of squares $Q_{1}, \ldots, Q_{\nu}$ with the property that every two consecutive squares $Q_{i}$ and $Q_{i+1}$ have an edge in common. Moreover, we say that a collection $\mathcal{B}$ of black squares $Q_{1}, \ldots, Q_{\nu}$ form a black island if the following conditions hold:

(i) $\mathcal{B}$ is connected. That is, for every $Q_{k}, Q_{l} \in B$ there is a sequence $Q_{i_{0}}, \ldots, Q_{i_{R}} \in \mathcal{B}$ with $i_{0}=k, i_{R}=l$ and the property that any two consecutive squares $Q_{i_{m}}, Q_{i_{m+1}}$ have at least one vertex in common.

(ii) $\mathcal{B}$ is maximal. That is, if a black square $Q$ has a point in common with an element of $\mathcal{B}$, then it belongs to $\mathcal{B}$.

(iii) $\mathcal{B}$ gets close to $\Sigma^{v}$. That is, there is a square $Q$ of $\mathcal{B}$ and a point $x \in \Sigma^{v}$ such that $\operatorname{dist}(x, Q) \leq 4 \sigma$.

The length of a black island and the length of a path are both defined as $\sigma$ multiplied by the number of squares forming the collection.

Next, given a black island $\mathcal{B}$ we can subdivide the set of squares not belonging to $\mathcal{B}$ (and hence denoted by $\mathcal{B}^{c}$ ) into connected components: two squares will belong to the same connected component if and only if there is a path connecting them which is completely contained in the complement $\mathcal{B}^{c}$. Of course, among these connected components there will be one and only one consisting of infinitely many squares. We will call it region exterior to the island. The remaining components will be called the regions enclosed by the island.

Given a black island $\mathcal{B}$, consider the collection $\mathcal{W}$ of all squares which lie on the exterior region and have at least one vertex in common with a square of the black island. By (i) and (ii), all these squares are white. We will call $\mathcal{W}$ the white island surrounding $\mathcal{B}$ (see Fig. 4). It is straightforward to check that any two squares of $\mathcal{W}$ can be connected by a path of squares contained in $\mathcal{W}$.

The following facts are clear:

- The white island enclosing a black island of length $\ell$ consists of at most $8 \ell / \sigma$ squares.

- The diameter of a region enclosed by a black island of length $\ell$ is at most $\ell$.

- If a point is at distance $2 \sqrt{2}(\ell+\sigma)$ from a square of a black island with length $\ell$, then it lies necessarily in the region exterior to the island.

8. We denote by $L_{\mathcal{B}}$ the sum of the lengths of all the black islands. The goal of this step is to provide an upper bound on $L_{\mathcal{B}}$. We begin by proving the following claim:

(C) For $\sigma$ sufficiently small, all black islands are entirely contained within the open set

$$
V \doteq B\left(\Sigma^{v}, 2 \rho_{1}\right) \cup\left(\bigcup_{\ell=1}^{m} B\left(\Gamma_{i(\ell)}, \rho_{2}\right)\right)
$$

Indeed, assume that the above claim were false. Then there is a sequence $\sigma_{k} \rightarrow 0$ and corresponding sequences of black islands $\mathcal{B}_{k}$ and points $x_{k} \in \mathcal{B}_{k} \backslash V$. With slight abuse of notation, we here denote by $\mathcal{B}_{k} \subset \mathbb{R}^{2}$ the union of the closed squares forming the island $\mathcal{B}_{k}$.

Each $\mathcal{B}_{k}$ is a compact connected set, whose diameter is uniformly bounded. Hence, by possibly taking a subsequence, we can assume that $\mathcal{B}_{k}$ converges to some compact set $K$ in the Hausdorff metric, while $x_{k} \rightarrow \bar{x} \notin V$. This limit set $K$ is clearly connected and contains $\bar{x}$. Moreover, by condition (iii) in the definition of black islands, $K$ intersects $\Sigma^{v}$ at some point $\bar{y}$.

We now claim that $\left(K \backslash \Sigma^{v}\right) \subseteq \Gamma$. Indeed, take any point $x \in K \backslash \Sigma^{v}$. Consider any $r<d\left(x, \Sigma^{v}\right) / 10$. For $k$ sufficiently large, the disk $B(x, r)$ contains a square of $\mathcal{B}_{k}$. On the other hand $\mathcal{B}_{k}$ also contains a point which lies outside $B(x, 2 r)$. Since $\mathcal{B}_{k}$ is connected, $B(x, 2 r)$ must contain at least $r /(\sqrt{2} \sigma)$ squares of $\mathcal{B}_{k}$ (see Fig. 5). Since each such square is black, we can estimate

$$
m_{1}(B(x, 2 r) \cap \Gamma) \geq \frac{r}{\sqrt{2} \sigma} \eta \sigma=\frac{\eta}{\sqrt{2}} r .
$$




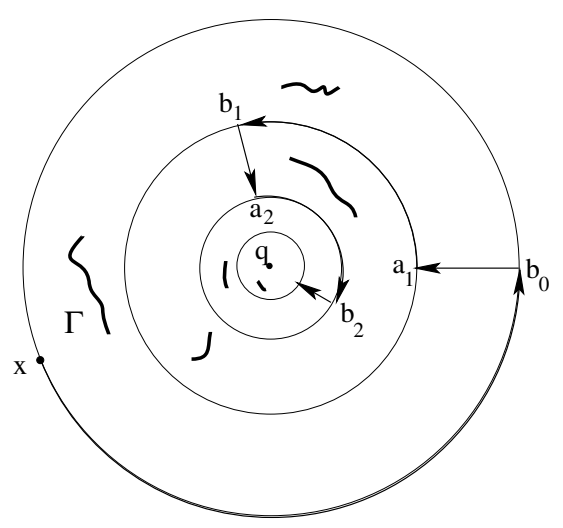

Figure 2. Construction of a path joining $q$ with points $x \in \partial B\left(q, r_{0}\right)$ without crossing $\Gamma$.

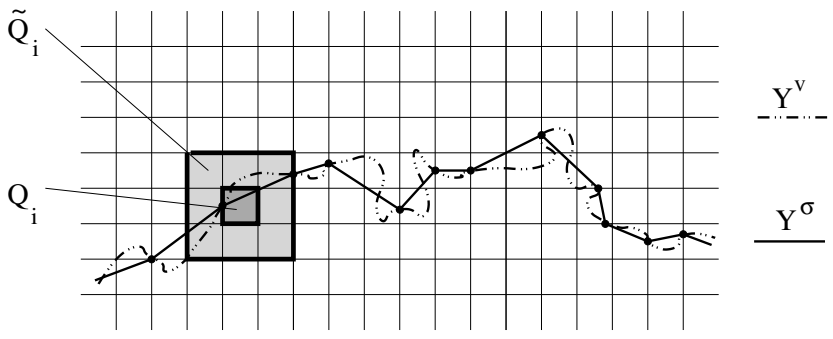

Figure 3. The grid of mesh $\sigma$, the shifted trajectory $Y^{v}$, and the polygonal trajectory $Y^{\sigma}$.

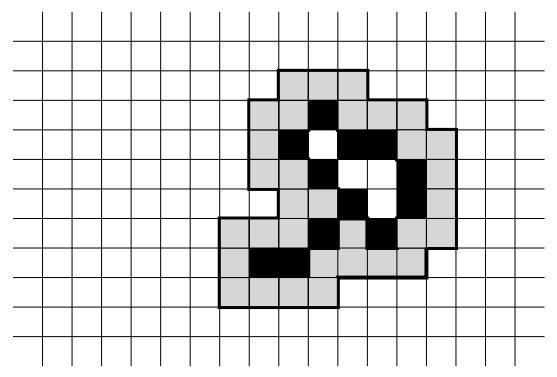

FiguRE 4. A black island $\mathcal{B}$ and the white island $\mathcal{W}$ surrounding $\mathcal{B}$.

Since $r>0$ is arbitrary, we conclude that the upper density of $\Gamma$ at $x$ satisfies $\theta^{*}(\Gamma, x)>0$, and hence $x \in \Gamma$, because the set $\Gamma$ is complete. This proves our claim.

Let $K_{x} \subseteq K$ be the closure of the connected component of $K \backslash \Sigma^{v}$ which contains the point $x$. Clearly $K_{x} \subseteq \Gamma$, because $\Gamma$ is complete. If $K_{x} \cap \Sigma^{v}=\emptyset$, then $K_{x}$ would be a proper connected component of the original set $K$. Since $K$ is connected, this would imply $K_{x}=K$ and hence $K_{x} \cap \Sigma^{v}=K \cap \Sigma^{v} \neq \emptyset$, reaching a contradiction. We thus conclude that $K_{x}$ is a connected component of $\Gamma$ which intersects $\Sigma^{v}$ and also contains a point $\bar{x} \notin V$. However, by the definition of the set $V$, no connected component $\Gamma_{i} \subseteq \Gamma$ exists with such properties. By contradiction, our claim (C) is thus proved.

Recalling (4.6) and (4.9)-(4.10), the total length of all black islands can now be estimated as

$$
L_{\mathcal{B}} \leq \frac{1}{\eta} m_{1}(\Gamma \cap V) \leq \frac{3 \delta}{\eta} .
$$




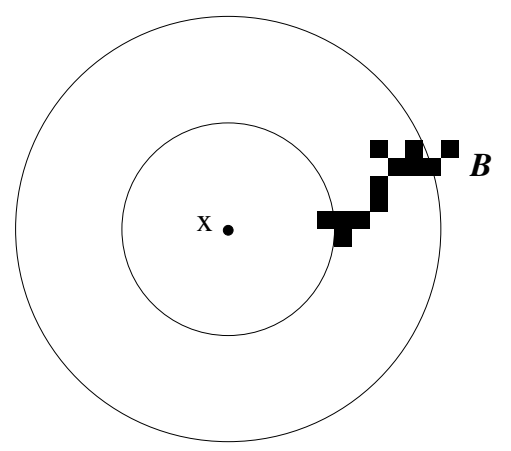

FiguRE 5. A chain of black squares joining a point in $B(x, r)$ to a point outside $B(x, 2 r)$ must contain at least $r / \sqrt{2} \sigma_{k}$ squares.

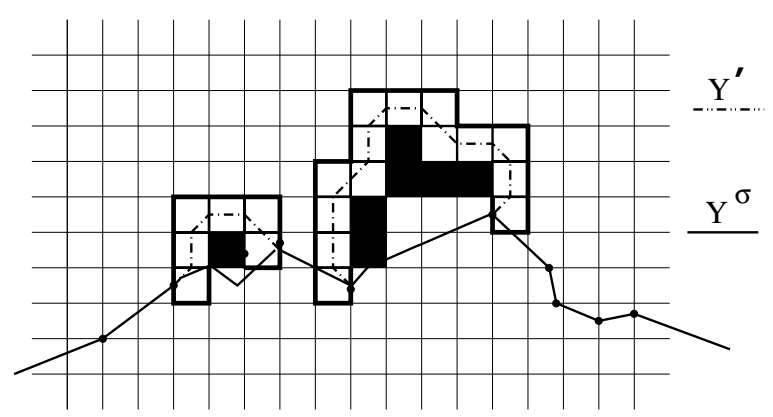

Figure 6. Construction of the curve $Y^{\prime}$. Compared with $Y^{\sigma}$, some portions have been removed and replaced by detours, to get around black islands.

9. Observing that each black island is entirely surrounded by a white island which is at most eight times longer, from (4.16) we deduce that the total length of all white islands surrounding black islands is

$$
L_{\mathcal{W}} \leq 8 L_{\mathcal{B}} \leq \frac{24 \delta}{\eta}
$$

We can now replace the polygonal $Y^{\sigma}$ by a second polygonal $Y^{\prime}$ living entirely on white squares. This is done as follows: whenever $Y^{\sigma}$ intersects a black chain, we insert a detour consisting of a polygonal curve living inside the surrounding white island, as shown in Figure 6. To fix the ideas, we choose each vertex of a detour exactly at the midpoint of some edge of the grid. In this way, each detour will be composed by segments having length either $\sigma$ or $\sigma / \sqrt{2}$.

Call $\xi_{0}^{\prime}, \xi_{1}^{\prime}, \ldots, \xi_{\nu}^{\prime}$ the intersections of the polygonal $Y^{\prime}$ with the vertical and horizontal lines of the grid. As in (4.14), we still have

$$
\left|\xi_{i+2}^{\prime}-\xi_{i+1}^{\prime}\right|+\left|\xi_{i+1}^{\prime}-\xi_{i}^{\prime}\right|+\left|\xi_{i}^{\prime}-\xi_{i-1}^{\prime}\right| \geq \sigma
$$

for every $i \in\{1, \ldots, \nu-2\}$. By (4.17), the length of this new polygonal is

$$
\left\|Y^{\prime}\right\| \leq\left\|Y^{\sigma}\right\|+\sqrt{2} L_{\mathcal{W}} \leq L+24 \sqrt{2} \delta
$$




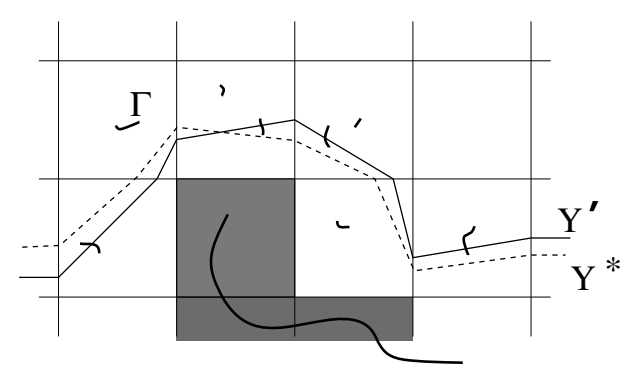

FiguRE 7. Given a polygonal $Y^{\prime}$ living entirely on white squares, one can construct an $\varepsilon$-close polygonal $Y^{*}$ which avoids the walls in $\Gamma$.

10. Since all segments of the polygonal $Y^{\prime}$ lie on white squares, by slightly shifting its vertices we can obtain a third polygonal $Y^{*}$ with vertices $\xi_{0}^{*}, \xi_{1}^{*}, \ldots, \xi_{\nu}^{*}$ which does not intersect $\Gamma$ (see Fig. 7). Indeed, using Lemma 4.1 inductively for $i=0,1, \ldots, \nu$, we can find points $\xi_{i}^{*}$ such that:

- Each $\xi_{i}^{*}$ lies on the same edge of the grid as $\xi_{i}^{\prime}$, and moreover $\left|\xi_{i}^{*}-\xi_{i}^{\prime}\right| \leq 2 \varepsilon \sigma$.

- None of the segments with endpoints $\xi_{i-1}^{*}, \xi_{i}^{*}$ intersects $\Gamma$.

Recalling (4.18), (4.19), and the first inequality in (4.5), by the above properties we conclude that the total length of this third polygonal is bounded by

$$
\begin{aligned}
\left\|Y^{*}\right\| & \leq\left\|Y^{\prime}\right\|+4 \varepsilon \sigma \nu \leq\left\|Y^{\prime}\right\|+4 \varepsilon \cdot 3\left\|Y^{\prime}\right\| \\
& \leq(1+12 \varepsilon)(L+24 \sqrt{2} \delta)<(1+12 \varepsilon)(L+\varepsilon) .
\end{aligned}
$$

11. We now have a polygonal curve $Y^{*}(\cdot)$ which does not intersect $\Gamma$. In this final step, we modify the beginning and the end of this path, so that it will start at $q_{0}$ and end at $q_{1}$, as required.

We observe that, assuming $\sigma>0$ small enough, the second inequality in (4.5) yields

$$
\sqrt{2}\left(\sigma+L_{\mathcal{W}}\right) \leq \frac{\min \left\{r_{0}, r_{1}\right\}}{2}
$$

Therefore (see Fig. 8) the initial point of the new polygonal $Y^{\prime}$ lies well inside the disc $B\left(q_{0}, r_{0}\right)$, while the terminal point of $Y^{\prime}$ lies inside $B\left(q_{1}, r_{1}\right)$. The following points are thus well defined:

$$
\begin{array}{ll}
p_{0} \doteq Y^{*}\left(s_{0}\right), & s_{0} \doteq \sup \left\{s \in[0,1] ; Y^{*}(s) \in B\left(q_{0}, r_{0}\right)\right\} \\
p_{1} \doteq Y^{*}\left(s_{1}\right), & s_{1} \doteq \inf \left\{s \in[0,1] ; \quad Y^{*}(s) \in B\left(q_{1}, r_{1}\right)\right\}
\end{array}
$$

By Lemma 4.3, there exists a path $Y_{0}(\cdot)$ connecting $q_{0}$ with $p_{0}$ without crossing $\Gamma$, having length $\leq(1+4 \pi) r_{0}$. Similarly, there exists a path $Y_{1}(\cdot)$ connecting $p_{1}$ with $q_{1}$ without crossing $\Gamma$, having length $\leq(1+4 \pi) r_{1}$.

Let $Y^{\sharp}$ be the path obtained as the concatenation of

- the path $Y_{0}$ from $q_{0}$ to $p_{0}$;

- the portion of $Y^{*}$ for $s \in\left[s_{0}, s_{1}\right]$, connecting $p_{0}$ with $p_{1}$;

- the path $Y_{1}$, connecting $p_{1}$ with $q_{1}$. 


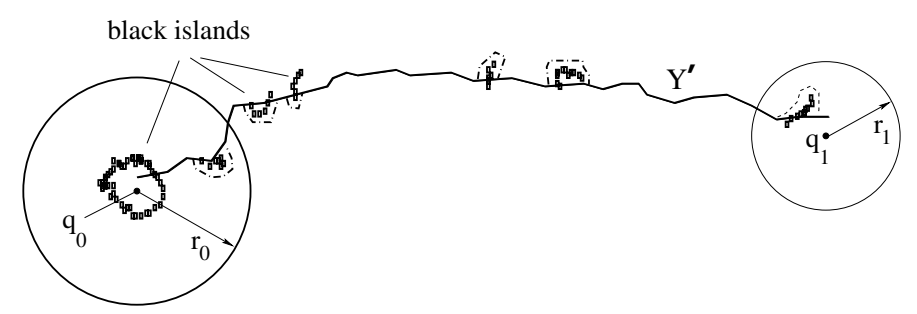

FiguRE 8 . The point $q_{0}$ may be entirely surrounded by a black island. However, since the radius $r_{0}$ is bigger than the total length of all black islands, the modified polygonal $Y^{\prime}$ still intersects the disc $B\left(q_{0}, r_{0}\right)$.

This path $s \mapsto Y^{\sharp}(s)$ connects $q_{0}$ with $q_{1}$ without crossing $\Gamma$. Moreover its length satisfies

$$
\begin{aligned}
\left\|Y^{\sharp}\right\| & \leq\left\|Y_{1}\right\|+\left\|Y^{*}\right\|+\left\|Y_{2}\right\| \leq(1+4 \pi) r_{0}+(1+12 \varepsilon)(L+\varepsilon)+(1+4 \pi) r_{1} \\
& \leq 2 \varepsilon(1+4 \pi) 2 \varepsilon+(1+12 \varepsilon)\left(\left|q_{1}-q_{0}\right|+\sum_{k \geq 1} m_{1}\left(\Gamma_{k}\right)+2 \varepsilon\right) .
\end{aligned}
$$

Since $\varepsilon>0$ can be chosen arbitrarily small, this achieves the proof of Lemma 4.4.

\section{Proof of the equivalence theorems}

In this section we provide a proof of Theorems 2.1 and 2.2, in several steps.

1. Let an admissible set $\Gamma \subset \mathbb{R}^{2}$ be given. Then by definition the strategy $t \mapsto \gamma(t)$ at $(2.2)$ is admissible. We claim that this strategy is also complete, according to the definition (H3) in Section 1. Indeed, let $x^{*}$ be a point of positive upper density for $\gamma(t)$. Then $x^{*} \in \Gamma$ because $\Gamma \supseteq \gamma(t)$ and $\Gamma$ is complete. Moreover, $x^{*} \in \overline{\gamma(t)} \subseteq \overline{R^{\Gamma}(t)}$. Hence $x^{*} \in \Gamma \cap \overline{R^{\Gamma}(t)}=\gamma(t)$, proving that each set $\gamma(t)$ is complete. Next let $x^{*} \in \Gamma \cap \overline{R^{\Gamma}\left(t_{n}\right)}$ for a decreasing sequence of times $t_{n} \downarrow t$. We need to show that $x^{*} \in \gamma(t)$. By assumption, there exists a sequence of trajectories $\tau \mapsto x_{n}(\tau)$ of the differential inclusion (1.1) which do not cross $\Gamma$ and satisfy $\left|x_{n}\left(t_{n}\right)-x^{*}\right|<1 / n$. Since the velocity sets $F(x)$ are uniformly bounded in a neighborhood of $x^{*}$, we have

$$
\left|x_{n}(t)-x^{*}\right| \leq\left|x_{n}(t)-x_{n}\left(t_{n}\right)\right|+\left|x_{n}\left(t_{n}\right)-x^{*}\right| \rightarrow 0
$$

as $n \rightarrow \infty$. Hence $x^{*} \in \overline{R^{\Gamma}(t)}$, completing the proof of our claim.

2. Since $\gamma(t) \subseteq \Gamma$ for all $t \geq 0$, the corresponding reachable sets trivially satisfy

$$
R^{\Gamma}(t) \subseteq R^{\gamma}(t) .
$$

To prove that equality holds, assume that there exists a point $y \in R^{\gamma}(t) \backslash R^{\Gamma}(t)$. We thus have a solution $\tau \mapsto x(\tau)$ of $(1.1)$ with $x(t)=y$, so that $x(\tau) \notin \gamma(\tau)$ for all $\tau \in[0, t]$ but $x\left(t^{\prime}\right) \in \Gamma$ for some $t^{\prime} \in[0, t]$. A contradiction is now derived as follows. Call $t^{*} \doteq \inf \left\{t^{\prime} \in[0, t] ; x\left(t^{\prime}\right) \in \Gamma\right\}$, and observe that

$$
x^{*} \doteq x\left(t^{*}\right)=\lim _{\tau \rightarrow t^{*}-} x(\tau) \in \overline{R^{\Gamma}\left(t^{*}\right)} .
$$

Two cases must be considered.

Case 1: $x\left(t^{*}\right) \in \Gamma$. This implies $x\left(t^{*}\right) \in \gamma\left(t^{*}\right)$, contradicting the assumption $x(\tau) \notin \gamma(\tau)$ for all $\tau \in[0, t]$. 


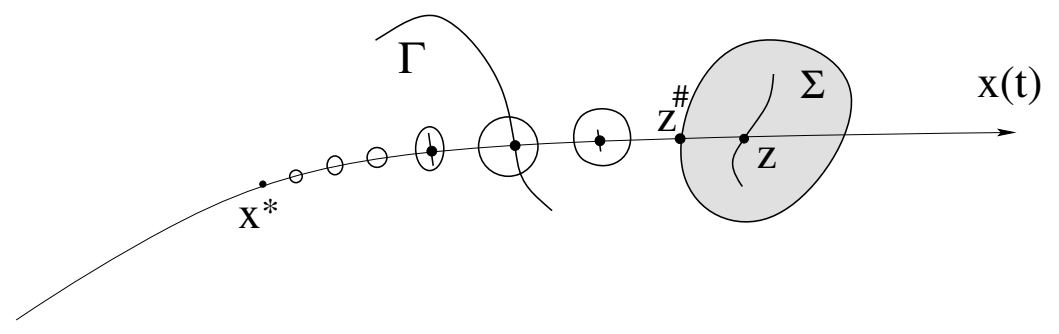

Figure 9. The construction used in the proof of Theorem 2.1.

Case 2: $x^{*} \doteq x\left(t^{*}\right) \notin \Gamma$. Notice that in this case, shown in Figure 9, we must have $t^{*}<t$. Since $R_{0}$ is open, $x_{0}$ lies in the interior of $R_{0}$. Hence we can choose $\varepsilon>0$ such that the map $\tau \mapsto \tilde{x}(\tau) \doteq x(\tau+\varepsilon)$ starts from a point $\tilde{x}(0)=x(\varepsilon) \in R_{0}$, and is thus an admissible trajectory, reaching the point $y$ at time $t-\varepsilon$. Consider the set $S_{\varepsilon}$ of points which can be reached at time $\varepsilon$, starting from $x^{*}$ and avoiding the set $\Gamma$. Namely

$$
\begin{aligned}
S_{\varepsilon} \doteq\{x(\varepsilon) ; & x(\cdot) \text { absolutely continuous, } x(0)=x^{*} \\
& \dot{x}(\tau) \in F(x(\tau)) \text { for a.e. } \tau \in[0, \varepsilon], \quad x(\tau) \notin \Gamma \text { for all } \tau \in[0, \varepsilon]\} .
\end{aligned}
$$

The above choice of $\varepsilon$ implies $S_{\varepsilon} \subseteq R^{\Gamma}\left(t^{*}\right)$. We shall achieve a contradiction by showing that

$$
\Gamma \cap \bar{S}_{\varepsilon} \cap\left\{x(\tau) ; \quad \tau \in\left[t^{*}, t\right]\right\} \neq \emptyset .
$$

By the assumption (A2), all velocity sets $F(x)$ contain a neighborhood of the origin. Hence there exists a radius $r^{*}>0$ such that $F(x) \supset B\left(0, r^{*}\right)$ for all $x$ in a neighborhood $V$ of $x^{*}$. We recall that $x^{*}$ lies in the closure of $\Gamma$ but is not a point of positive upper density for $\Gamma$. Using Lemma 4.3 we can find a radius $\rho>0$ small enough such that the circumference $\partial B\left(x^{*}, \rho\right)=\left\{x ;\left|x-x^{*}\right|=\rho\right\}$ does not intersect $\Gamma$, and is entirely contained in the reachable set $S_{\varepsilon}$. Moreover, by choosing $\rho>0$ small enough, we can also assume that $B\left(x^{*}, \rho\right) \subset V$ and

$$
\rho+m_{1}\left(\Gamma \cap B\left(x^{*}, \rho\right)\right)<\frac{\varepsilon r^{*}}{2}
$$

Using Lemma 4.4 we now prove an important property of the set $S_{\varepsilon}$.

(P) For every $y \in B\left(x^{*}, \rho\right)$ one has $y \in S_{\varepsilon}$ if and only if the two points $y$ and $x^{*}$ lie in the same connected component of $\mathbb{R}^{2} \backslash\left(\Gamma \cap B\left(x^{*}, \rho\right)\right)$.

Indeed, if $y$ and $x^{*}$ lie in the same connected component, by Lemma 4.4 there exists a path $s \mapsto Y^{\sharp}(s)$ of length

$$
\left\|Y^{\sharp}\right\| \leq\left|y-x^{*}\right|+m_{1}\left(\Gamma \cap B\left(x^{*}, \rho\right)\right)+\frac{\varepsilon r^{*}}{2}<\varepsilon r^{*}
$$

connecting $x^{*}$ with $y$ without crossing the set $\Gamma \cap B\left(x^{*}, \rho\right)$. Call $\pi: \mathbb{R}^{2} \mapsto \bar{B}\left(x^{*}, \rho\right)$ the perpendicular projection onto the closed disc centered at $x^{*}$ with radius $\rho$. Then the projected path $s \mapsto \pi Y^{\sharp}(s)$ has length $\left\|\pi Y^{\sharp}\right\| \leq$ $\left\|Y^{\sharp}\right\|<\varepsilon r^{*}$ and connects $x^{*}$ with $y$ without crossing $\Gamma$, because $\Gamma \cap \partial B\left(x^{*}, \rho\right)=\emptyset$. Since $B\left(0, r^{*}\right) \subseteq F(x)$, there exists a solution of the differential inclusion $\dot{x} \in F(x)$ which starts from $x^{*}$, follows the path $\pi Y^{\sharp}$, and reaches $y$ within time $\varepsilon$. Hence $x \in S_{\varepsilon}$. This proves one part of the statement $(\mathrm{P})$. The converse is trivial.

Next, choose any point

$$
z \in \Gamma \cap B\left(x^{*}, \rho\right) \cap\left\{x(\tau) ; \quad \tau \in\left[t^{*}, t\right]\right\}
$$

If $z \in \bar{S}_{\varepsilon} \subseteq \overline{R\left(t^{*}\right)}$ we are done. Otherwise, define $\Sigma$ as the open connected component of $\mathbb{R}^{2} \backslash \overline{S_{\varepsilon}}$ containing $z$. Observe that $\Sigma \subset B\left(x^{*}, \rho\right)$. We claim that every boundary point $w \in \partial \Sigma$ must lie in $\Gamma$. Indeed, assume 
on the contrary that $w \in \partial \Sigma \backslash \Gamma$. Then by the completeness assumption it follows that $w$ is not a point of positive upper density for $\Gamma$. Hence we can find a radius $r>0$ such that

$$
\Gamma \cap \partial B(w, r)=\emptyset, \quad x^{*}, z \notin \bar{B}(w, r) \subset B\left(x^{*}, \rho\right)
$$

By the above construction it is clear that the intersection $\partial B(w, r) \cap S_{\varepsilon}$ is nonempty. Relying on the property (P), we conclude that $\partial B(w, r) \subset S_{\varepsilon}$, hence $w$ and $z$ lie on distinct connected components of $\mathbb{R}^{2} \backslash \bar{S}_{\varepsilon}$, a contradiction. Since $x^{*} \notin \Sigma$ while $z \in \Sigma$, there exists a time $t^{\sharp} \in\left[t^{*}, t\right]$ such that $z^{\sharp} \doteq x\left(t^{\sharp}\right) \in \partial \Sigma$. By the previous argument, $z^{\sharp} \in \Gamma$. Moreover, by construction $z^{\sharp} \in \partial \Sigma \subseteq \partial S_{\varepsilon} \subseteq \overline{R^{\Gamma}\left(t^{*}\right)}$.

Summarizing: In both Cases 1 and 2 we showed the existence of a time $t^{\sharp} \in\left[t^{*}, t\right]$ such that $x\left(t^{\sharp}\right) \in \Gamma \cap \overline{R^{\Gamma}\left(t^{\sharp}\right)}$, hence $x\left(t^{\sharp}\right) \in \gamma\left(t^{\sharp}\right)$. This contradiction shows that

$$
R^{\Gamma}(t)=R^{\gamma}(t) \quad \text { for all } t \geq 0
$$

3. Next, consider a complete, admissible strategy $t \mapsto \gamma(t)$ and define the set $\Gamma$ according to (2.4). To analyze the corresponding reachable sets $R^{\Gamma}(t)$ and $R^{\gamma}(t)$, we introduce a countable sequence of admissible strategies $\gamma_{n}(\cdot)$, constructed as follows. For notational convenience, set $R^{\gamma}(t) \doteq R_{0}$ for $t \leq 0$. Consider the minimum time functions

$$
T^{R}(x) \doteq \inf \left\{t \geq-1 ; x \in R^{\gamma}(t)\right\}, \quad T^{\gamma}(x) \doteq \inf \{t \geq 0 ; x \in \gamma(t)\},
$$

defined on the sets $R_{\infty}^{\gamma}$ and $\gamma_{\infty}$, respectively. Notice that $T^{\gamma}(x)$ is actually a minimum, because of (1.4). Moreover, according to our convention, $T^{R}(x)=-1$ for all $x \in R_{0}$. We then define

$$
\begin{gathered}
\Gamma_{n} \doteq \text { completion of }\left\{x \in \gamma_{\infty} ; T^{\gamma}(x)<T^{R}(x)+\frac{1}{n}\right\} \\
\gamma_{n}(t) \doteq \gamma(t) \cap \Gamma_{n} .
\end{gathered}
$$

Roughly speaking, the wall $\gamma_{n}(t)$ is obtained from $\gamma(t)$ by removing some useless portions, i.e. points already reached by the fire at an earlier time.

Since $\gamma(\cdot)$ is a complete admissible strategy and each $\Gamma_{n}$ is a complete rectifiable set, it is clear that each $\gamma_{n}(\cdot)$ is a complete admissible strategy as well. We now prove the inclusions

$$
R^{\gamma}(t) \subseteq R^{\gamma_{n}}(t) \subseteq R^{\gamma}(t) \cup \gamma(t) \cup \partial R_{0} \quad \text { for all } t \geq 0, \quad n \geq 1
$$

Since $\gamma_{n}(t) \subseteq \gamma(t)$ for every $n \geq 1$ and $t \geq 0$, the first inclusion in (5.7) is clear.

To prove the second inclusion, consider any point $y \in R^{\gamma_{n}}(t) \backslash \gamma(t)$. By assumption, there exists a trajectory of (1.1) $\tau \mapsto x(\tau) \notin \gamma_{n}(\tau)$, with $x(t)=y$. If $x(\tau) \notin \gamma(\tau)$ for all $\tau \in[0, t]$, then $x(t) \in R^{\gamma}(t)$ and we are done. Otherwise, calling

we consider three cases.

$$
\tau^{*} \doteq \sup \{\tau \in[0, t] ; \quad x(\tau) \in \gamma(\tau)\}
$$

Case 1: $x^{*}=x\left(\tau^{*}\right) \in R_{0}$. Notice that this happens, in particular, if $\tau^{*}=0$. In this case, since $R_{0}$ is open, we can find $\varepsilon>0$ such that $x\left(\tau^{*}+\varepsilon\right) \in R_{0}$. Then the path

$$
\hat{x}(\tau)=\left\{\begin{aligned}
x\left(\tau+\tau^{*}+\varepsilon\right) & \text { if } \tau \in\left[0, t-\tau^{*}-\varepsilon\right], \\
y & \text { if } \tau \in\left[t-\tau^{*}-\varepsilon, t\right]
\end{aligned}\right.
$$

satisfies $\hat{x}(\tau) \notin \gamma(\tau)$ for all $\tau \in[0, t]$ and reaches the point $y$ at time $t$. Therefore $y \in R^{\gamma}(t)$. 
Case 2: $\tau^{*}>0$ and $x^{*} \doteq x\left(\tau^{*}\right) \in \gamma\left(\tau^{*}\right)$. In this case, since $x^{*} \notin \gamma_{n}\left(\tau^{*}\right)$, we must have

$$
T^{R}\left(x^{*}\right) \leq T^{\gamma}\left(x^{*}\right)-\frac{1}{n} \leq \tau^{*}-\frac{1}{n}
$$

therefore $x^{*} \in R^{\gamma}\left(s^{*}\right)$ for some $s^{*}<\tau^{*}$. Hence there exists a trajectory of (1.1), say $s \mapsto \tilde{x}(s) \notin \gamma(s)$, with $\tilde{x}\left(s^{*}\right)=x^{*}$. Calling

$$
\hat{x}(\tau) \doteq\left\{\begin{array}{rll}
\tilde{x}(\tau) & \text { if } & \tau \in\left[0, s^{*}\right], \\
x\left(\tau-\tau^{*}+s^{*}\right) & \text { if } \tau \in\left[s^{*}, t-\tau^{*}+s^{*}\right], \\
x^{*} & \text { if } \tau \in\left[t-\tau^{*}+s^{*}, t\right],
\end{array}\right.
$$

we obtain a new trajectory which reaches $y$ at time $t$ and satisfies $\hat{x}(\tau) \notin \gamma(\tau)$ for all $\tau \in[0, t]$. Hence $y \in R^{\gamma}(t)$.

Case 3: $x^{*} \doteq x\left(\tau^{*}\right) \notin \gamma\left(\tau^{*}\right) \cup \bar{R}_{0}$. In this case, observing that $x^{*}$ is not a point of density for $\gamma\left(\tau^{*}\right)$, using Lemma 4 and the assumption (A2) on $F$, we can find sufficiently small radii $r^{*}, \rho>0$ such that the following holds:

(i) $B\left(x^{*}, 2 \rho\right) \cap R_{0}=\emptyset$ and $\partial B\left(x^{*}, \rho\right) \cap \gamma\left(\tau^{*}\right)=\emptyset$.

(ii) Every point $z \in \partial B\left(x^{*}, \rho\right)$ can be connected to $x^{*}$ by a path with length $<\left(r^{*} / 2\right) \cdot \min \left\{\tau^{*}, 1 / n\right\}$ without crossing $\gamma\left(\tau^{*}\right)$

(iii) For every $x \in \bar{B}\left(x^{*}, \rho\right)$ one has $F(x) \supseteq B\left(0, r^{*}\right)$.

Since $x\left(\tau^{\prime}\right) \notin \gamma_{n}\left(\tau^{\prime}\right)$, by the definition of $\gamma_{n}$ there exists a second solution of $(1.1) s \mapsto \tilde{x}(s) \notin \gamma(s)$, starting from a point $\tilde{x}(0) \in R_{0}$ and reaching the point $\tilde{x}\left(s^{\prime}\right)=x\left(\tau^{\prime}\right)$ at some earlier time

$$
s^{\prime}<\tau^{\prime}-\frac{1}{2} \min \left\{\tau^{*}, \frac{1}{n}\right\}
$$

By (i) we have $\tilde{x}(0) \notin \bar{B}\left(x^{*}, \rho\right)$. Hence, to reach the point $x^{*}$, this trajectory must cross the circumference $\partial B\left(x^{*}, \rho\right)$ at some time $s^{\prime \prime}<s^{\prime}$.

By (ii), there exists a path connecting $\tilde{x}\left(s^{\prime \prime}\right) \in \partial B\left(x^{*}, \rho\right)$ with $x^{*}$ without $\operatorname{crossing} \gamma\left(\tau^{*}\right)$. Call $\xi:[0, \eta] \mapsto$ $\mathbb{R}^{2} \backslash \gamma\left(\tau^{*}\right)$ this path, parameterized by arc length. Observe that its total length is $\eta<\left(r^{*} / 2\right) \cdot \min \left\{\tau^{*}, 1 / n\right\}$. Because of (iii), this path can be traversed by a trajectory of the differential inclusion $\dot{x} \in F(x)$ within time $\eta / r^{*}$. By a concatenation, we thus obtain a path

$$
\hat{x}(\tau) \doteq\left\{\begin{aligned}
\tilde{x}(\tau) & \text { if } \quad \tau \in\left[0, s^{\prime \prime}\right], \\
\xi\left(\left(\tau-s^{\prime \prime}\right) r^{*}\right) & \text { if } \tau \in\left[s^{\prime \prime}, s^{\prime \prime}+\left(\eta / r^{*}\right)\right], \\
x^{*} & \text { if } \tau \in\left[s^{\prime \prime}+\left(\eta / r^{*}\right), t\right],
\end{aligned}\right.
$$

reaching the point $y$ without crossing the sets $\gamma(\tau)$. Once again, this proves $y \in R^{\gamma}(t)$.

Together, the above three cases yield (5.7). Observing that $R^{\gamma_{n}}(t) \subseteq \overline{R^{\gamma_{n}}(t) \backslash \gamma(t)}$ and recalling the assumption $(\mathrm{A} 1)$ on $\partial R_{0}$, from $(5.7)$ we deduce

$$
\overline{R^{\gamma_{n}}(t)}=\overline{R^{\gamma}(t)}, \quad \quad m_{2}\left(R^{\gamma_{n}}(t)\right)=m_{2}\left(R^{\gamma}(t)\right) \quad \text { for all } t \geq 0, n \geq 1 .
$$

Remark. If the open set $R_{0}$ is connected, or it has a Lipschitz boundary, then one could actually prove that $R^{\gamma}(t)=R^{\gamma_{n}}(t) \backslash \gamma(t)$. In any case, the weaker statement (5.7) suffices for our purpose.

4. We now recall the basic lower semicontinuity result proved in [4]. Let $\left(\gamma_{n}\right)_{n \geq 1}$ be a sequence of complete admissible strategies. For every $t \geq 0$ we can order the connected components of $\gamma_{n}(t)$ according to decreasing length, namely

$$
\gamma_{n}(t)=\left(\gamma_{n 1}(t) \cup \gamma_{n 2}(t) \cup \ldots\right) \cup \gamma_{n 0}(t)
$$


Here $\gamma_{n 0}(t)$ is a totally disconnected set, while for $j \geq 1$ the sets $\gamma_{n j}(t)$ are compact connected components, with lengths

$$
m_{1}\left(\gamma_{n 1}(t)\right) \geq m_{1}\left(\gamma_{n 2}(t)\right) \geq \ldots>0
$$

By a diagonal procedure one can select a subsequence, which for simplicity will still be called $\left(\gamma_{n}\right)_{n \geq 1}$, such that for every $j \geq 1$ and for every rational time $t \in \mathbf{Q}$ the corresponding sequence of compact sets converges in the Hausdorff metric:

$$
\gamma_{n j}(t) \rightarrow \gamma_{j}^{\sharp}(t) \quad \text { as } n \rightarrow \infty,
$$

for some compact sets $\gamma_{j}^{\sharp}(t)$. At rational times $t \in \mathbf{Q}$ we define

$$
\gamma^{\sharp}(t) \doteq \text { completion of }\left(\bigcup_{j \in I(t)} \gamma_{j}^{\sharp}(t)\right),
$$

where $I(t)$ is the set of indices $j$ such that $\liminf _{n \rightarrow \infty} m_{1}\left(\gamma_{n, j}(t)\right)>0$. For arbitrary times $t \geq 0$ we then define

$$
\gamma^{*}(t) \doteq \bigcap_{s>t, s \in \mathbf{Q}} \gamma^{\sharp}(s) .
$$

As proved in [4], under the assumptions (A1)-(A3) the strategy $\gamma^{*}(\cdot)$ is admissible and complete. The corresponding reachable sets satisfy

$$
m_{2}\left(R^{\gamma^{*}}(t)\right) \leq \liminf _{n \rightarrow \infty} m_{2}\left(R^{\gamma_{n}}(t)\right)
$$

Moreover, for every $t \geq 0$ and $y \in \overline{R^{\gamma^{*}}(t)}$, there exists a sequence of admissible trajectories of (1.1), say $\tau \mapsto x_{n}(\tau) \notin \gamma_{n}(\tau)$, with

$$
x_{n}(t) \rightarrow y \quad \text { as } n \rightarrow \infty .
$$

In [4], assuming that $\left(\gamma_{n}\right)_{n \geq 1}$ is a minimizing sequence for the cost functional (1.6), these lower semicontinuity results were used to prove that $\gamma^{*}$ is actually an optimal strategy. In the present setting, where the strategies $\gamma_{n}$ are defined by (5.5)-(5.6), from (5.8) it follows

$$
\overline{R^{\gamma^{*}}(t)}=\overline{R^{\gamma}(t)}, \quad m_{2}\left(R^{\gamma^{*}}(t)\right)=m_{2}\left(R^{\gamma}(t)\right) \quad \text { for all } t \geq 0 .
$$

5. Our next goal is to compare the strategy $\gamma^{*}(\cdot)$ with

$$
\gamma^{\dagger}(t) \doteq \Gamma \cap \gamma(t)
$$

Since $\Gamma$ is complete, it is clear that $\gamma^{\dagger}$ is also a complete, admissible strategy. We claim that

$$
\gamma^{*}(t) \subseteq \gamma^{\dagger}(t) \quad \text { for all } t \geq 0 .
$$

Indeed, consider any compact connected component $\gamma_{j}^{\sharp}(t)$ with positive length, as in (5.9). Since the sets $\Gamma_{n}$ are decreasing and complete, one must have $\gamma_{j}^{\sharp}(t) \subseteq \Gamma_{n} \cap \gamma(t)$ for every $n \geq 1$. Since $m_{1}\left(\bigcap_{n \geq 1} \Gamma_{n} \backslash \Gamma\right)=0$ and $\Gamma$ is complete, this yields $\gamma_{j}^{\sharp}(t) \subseteq \Gamma \cap \gamma(t)$. Observing that $t \mapsto \gamma^{*}(t)$ is the smallest complete strategy which contains all the sets $\gamma_{j}^{\sharp}(t)$ for each rational time $t$, we obtain (5.12).

By (5.11) it now follows

$$
\overline{R^{\gamma}(t)} \subseteq \overline{R^{\gamma^{\dagger}}(t)} \subseteq \overline{R^{\gamma^{*}}(t)}=\overline{R^{\gamma}(t)} \quad \text { for all } t \geq 0
$$

showing that the closures of all these reachable sets are equal. 
From the above relations we obtain

$$
\begin{gathered}
\overline{R^{\Gamma}(t)} \subseteq \overline{R^{\gamma^{\dagger}}(t)}=\overline{R^{\gamma}(t)} \\
\Gamma \cap \overline{R^{\Gamma}(t)} \subseteq \Gamma \cap \overline{R^{\gamma}(t)} \subseteq \Gamma \cap \gamma(t) .
\end{gathered}
$$

Following (2.2), we now define the strategy $\tilde{\gamma}(t) \doteq \Gamma \cap \overline{R^{\Gamma}(t)}$. By (5.15) one has

$$
\int_{\tilde{\gamma}(t)} \psi \mathrm{d} m_{1} \leq \int_{\gamma(t)} \psi \mathrm{d} m_{1} \leq t,
$$

showing that the set $\Gamma$ is admissible. Moreover, the analysis in steps $\mathbf{1}-\mathbf{2}$ shows that

$$
R^{\Gamma}(t)=R^{\tilde{\gamma}}(t) \quad \text { for all } t \geq 0
$$

This is indeed the identity proved at $(5.4)$, where the strategy $\gamma(\cdot)$ is now called $\tilde{\gamma}(\cdot)$.

6. Relying on the previous analysis, we now easily obtain a proof of Theorem 2.1 .

If the complete rectifiable set $\Gamma$ is admissible, and $R^{\Gamma}(t) \subseteq B_{r}$ for all $t \geq 0$, then by (5.4) the strategy $\gamma(\cdot)$ defined at (2.2) yields $R^{\gamma}(t)=R^{\Gamma}(t) \subseteq B_{r}$.

Vice versa, if the admissible strategy $\gamma(\cdot)$ solves the blocking problem (BP1), then the corresponding set $\Gamma$ in (2.4) is admissible and, by (5.14), $\overline{R^{\Gamma}(t)} \subseteq \overline{R^{\gamma}(t)}$ for all $t \geq 0$. Hence $\Gamma$ solves the blocking problem (BP2).

7. To prove Theorem 2.2, some additional notation will be useful. Given an admissible strategy $\gamma$, we let $\Gamma(\gamma)$ be the corresponding set, as in (2.4). On the other hand, given an admissible set $\Gamma$, we define the strategy $\gamma(\Gamma)$ according to $(2.2)$.

Let $J(\gamma)$ and $J(\Gamma)$ be the cost functionals defined at (1.6) and (2.3), respectively. From the previous analysis it follows

$$
J(\Gamma(\gamma)) \leq J(\gamma), \quad J(\gamma(\Gamma)) \leq J(\gamma)
$$

Let now $\gamma^{*}(\cdot)$ be an optimal strategy for the minimization problem (OP1). Then, for any admissible set $\widetilde{\Gamma}$, we have

$$
J\left(\Gamma\left(\gamma^{*}\right)\right) \leq J\left(\gamma^{*}\right) \leq J(\gamma(\widetilde{\Gamma})) \leq J(\widetilde{\Gamma})
$$

Therefore the strategy $\Gamma\left(\gamma^{*}\right)$ is optimal for (OP2).

On the other hand, assume that the set $\Gamma^{*}$ provides an optimal solution to the minimization problem (OP2). Then, for any admissible strategy $\tilde{\gamma}(\cdot)$, we have

$$
J\left(\gamma\left(\Gamma^{*}\right)\right) \leq J\left(\Gamma^{*}\right) \leq J(\Gamma(\tilde{\gamma})) \leq J(\tilde{\gamma}) .
$$

Therefore the strategy $\gamma\left(\Gamma^{*}\right)$ is optimal for $(\mathrm{OP} 1)$.

\section{A NUMERICAL ALGORITHM}

According to Theorem 2.2, the optimization problems (OP1) and (OP2) are equivalent. Motivated by this result, in this section we develop a numerical algorithm to solve (OP2). This problem is easier to handle, because it involves one single rectifiable set $\Gamma$, rather than a family of sets $\gamma(t)$.

For simplicity, we shall restrict our analysis to the case where the minimum time function does not depend on the set $\Gamma$. More precisely, given the differential inclusion (1.1), recalling (1.2) we define

$$
T(x) \doteq \inf \{t \geq 0 ; \quad x \in R(t)\}
$$



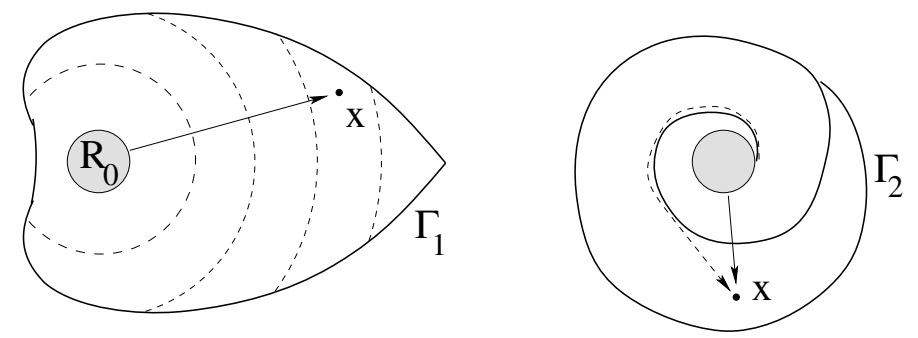

Figure 10. Consider the isotropic case, where the fire starts from the unit disc and propagates with unit speed in all directions. Then the assumption (6.3) is satisfied for the wall $\Gamma_{1}$ on the left, but not for the rectifiable set $\Gamma_{2}$ on the right.

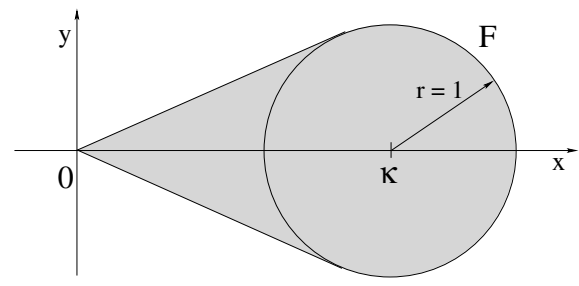

Figure 11. The set $F$ of propagation velocities, defined at (6.6).

Clearly, $T(x)$ is the minimum time needed by the fire to reach the point $x$ starting from $R_{0}$. Similarly, given a complete rectifiable set $\Gamma$, recalling (2.1) we define

$$
T^{\Gamma}(x) \doteq \inf \left\{t \geq 0 ; x \in R^{\Gamma}(t)\right\} .
$$

Throughout the following, our search of optimal solutions to (OP2) will be restricted within the class of rectifiable sets $\Gamma$ with the property that

$$
x \in R_{\infty}^{\Gamma} \quad \Longrightarrow \quad T^{\Gamma}(x)=T(x) .
$$

Intuitively this means that, if the point $x$ is reachable from $R_{0}$ without crossing $\Gamma$, then the fastest path from $R_{0}$ to $x$ stays away from $\Gamma$ (see Fig. 10). This assumption is usually met by the optimal solution, in practical situations.

The optimization problem we consider is:

$$
\begin{gathered}
\text { minimize: } \quad\left\{m_{2}\left(R_{\infty}^{\Gamma}\right)+\lambda \cdot m_{1}(\Gamma)\right\}=\begin{array}{r}
\text { area of the burned region] } \\
+\lambda \cdot \text { [length of the wall] },
\end{array} \\
\text { subject to: } \quad m_{1}\left(\Gamma \cap \overline{R^{\Gamma}(t)}\right) \leq \sigma t \quad \text { for all } t \geq 0 .
\end{gathered}
$$

Here $\lambda \geq 0$ and $\sigma>0$ are fixed constants.

Our numerical algorithm will be tested on two problems where an analytic solution is already known [3,6]. The first is the isotropic case, where the fire starts from the unit disc and propagates with unit speed in all directions. In the second case, studied in [6], the initial set is again the unit disc $R_{0}=B_{1}$, but as velocity sets we take

$$
F(x) \equiv F \doteq\left\{\left(\theta\left(\kappa+y_{1}\right), \theta y_{2}\right) ; \theta \in[0,1], \quad y_{1}^{2}+y_{2}^{2} \leq 1\right\},
$$

where $\kappa>1$ is a fixed positive number. Because of the shape of this set, this non-isotropic problem will be called the "ice-cream cone" case, see Figure 11. 


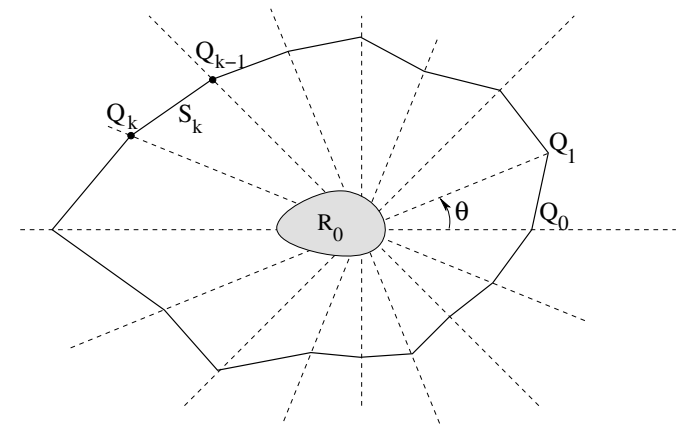

Figure 12. A polygonal approximation the the curve $\Gamma$.

In our algorithm, as shown in Figure 12, the curve $\Gamma$ is approximated by a polygonal $\mathcal{P}$ whose vertices lie on a fixed family of rays, originating from a given point in $\mathbb{R}^{2}$. We start with an initial guess $\mathcal{P}_{0}$, satisfying a discretized version of the admissibility conditions (6.5). By a suitable iteration procedure, we then construct a sequence of polygonals $\mathcal{P}_{1}, \mathcal{P}_{2}, \mathcal{P}_{3}, \ldots$ which are all admissible, and such that

$$
m_{2}\left(R_{\infty}^{\mathcal{P}_{0}}\right) \geq m_{2}\left(R_{\infty}^{\mathcal{P}_{1}}\right) \geq m_{2}\left(R_{\infty}^{\mathcal{P}_{2}}\right) \geq \ldots
$$

The effectiveness of this limiting process is evaluated by comparing numerical results with the exact solutions derived in $[3,6]$.

Finally, some comments about the search directions and drawback of the algorithms are given in the last section. To reduce the running cost, a modification of the line-search algorithm for the isotropic case is proposed.

\subsection{Polygonal approximations}

To define a family of polygonal approximations, we fix an integer $n \geq 3$ and consider closed polygonal curves $\mathcal{P}$ having vertices at points $Q_{k}=\left(\rho_{k} \cos k \theta, \rho_{k} \sin k \theta\right), 1 \leq k \leq n$. Here $\rho_{0}, \ldots, \rho_{n}$ are positive numbers with $\rho_{0}=\rho_{n}$, while $\theta \doteq 2 \pi / n$. We call $S_{k}$ the edge of the polygonal joining $Q_{k-1}$ with $Q_{k}$. Its length is computed by

$$
\left\|S_{k}\right\|=\sqrt{\rho_{k}^{2}+\rho_{k-1}^{2}-2 \cos \theta \rho_{k} \rho_{k-1}} .
$$

Setting $\vec{\rho} \doteq\left(\rho_{1}, \ldots, \rho_{n}\right)$, the area enclosed by the polygonal is computed as

$$
A(\vec{\rho})=\frac{1}{2} \sin \theta \cdot \sum_{k=1}^{n} \rho_{k} \rho_{k-1}
$$

while the total length is

$$
L(\vec{\rho})=\sum_{k=1}^{n} \sqrt{\rho_{k}^{2}+\rho_{k-1}^{2}-2 \cos \theta \rho_{k} \rho_{k-1}} .
$$

Moreover, the minimum time needed by the fire to reach some point on the segment $S_{k}$ is defined as

$$
T\left(S_{k}\right) \doteq \inf \left\{T(x) ; x \in S_{k}\right\}
$$

A discrete approximation to the constrained optimization problem (6.4)-(6.5) is given by

$$
\min _{\vec{\rho}}\{A(\vec{\rho})+\lambda \cdot L(\vec{\rho})\}
$$


subject to the family of constraints

$$
\sum_{k=1}^{m}\left\|S_{i_{k}}\right\|-\sigma \cdot T\left(S_{i_{m}}\right) \leq 0 \quad \text { for all } \quad m=1,2, \ldots, n .
$$

Here $\left(i_{1}, i_{2}, \ldots, i_{n}\right)$ is some permutation of the indices $(1,2, \ldots, n)$ such that

$$
T\left(S_{i_{1}}\right) \leq T\left(S_{i_{2}}\right) \leq \ldots \leq T\left(S_{i_{n}}\right) .
$$

We denote by $\mathcal{F}_{\vec{\rho}}$ the collection of all such possible permutations, for a given $\vec{\rho}$. Observe that, if the constraints (6.8) are satisfied for one permutation $\alpha \in \mathcal{F}_{\vec{\rho}}$, then they are necessarily satisfied for every permutation $\beta \in \mathcal{F}_{\vec{\rho}}$.

\subsection{A line-search algorithm}

For a fixed permutation $\alpha=\left(i_{1}^{\alpha}, i_{2}^{\alpha}, \ldots, i_{n}^{\alpha}\right)$, let $\Omega_{\alpha}$ be the set of $n$-tuples $\vec{\rho}=\left(\rho_{1}, \ldots, \rho_{n}\right)$ for which the following collection of constraints is satisfied:

$$
\begin{array}{rlr}
c_{m}^{\alpha}(\vec{\rho}) \doteq \sum_{j=1}^{m}\left\|S_{i_{j}^{\alpha}}\right\|-\sigma \cdot T\left(S_{i_{m}^{\alpha}}\right) \leq 0, & 1 \leq m \leq n, \\
c_{n+m}^{\alpha}(\vec{\rho}) \doteq T\left(S_{i_{m}^{\alpha}}\right)-T\left(S_{i_{m+1}^{\alpha}}\right) \leq 0, & 1 \leq m \leq n-1 .
\end{array}
$$

Moreover, we call $\Omega \doteq \bigcup_{\alpha} \Omega_{\alpha}$ the admissible set, on which the minimum in (6.7) is sought.

Our algorithm combines different steps. Given a permutation $\alpha$, and an initial guess $\vec{\rho}_{0} \in \Omega_{\alpha}$ we consider a minimization problem, restricted to the closed set $\Omega_{\alpha}$. By iterated line searches we obtain a local minimizer $\vec{\rho}_{*} \in \Omega_{\alpha}$. Next, for every $\beta \in \mathcal{F}_{\vec{\rho}_{*}}$, we check whether $\vec{\rho}_{*}$ is also a local minimizer restricted to $\Omega_{\beta}$; if not, we carry out a new minimization procedure on the closed set $\Omega_{\beta}$, starting from $\vec{\rho}_{*}$. When an approximate local minimum is found, we double the number of mesh points, and restart the procedure. A more precise formulation of the algorithm is given below.

Step 1. Choose an integer $n=n_{0} \geq 3$ as the initial number of vertices of the polygon. Pick a feasible $n_{0}$-tuple $\vec{\rho}_{0}$ as initial guess. Choose a permutation $\alpha_{0} \in \mathcal{F}_{\overrightarrow{\rho_{0}}}$. Go to Step 2.

Step 2. Let a polygonal $\vec{\rho}_{j}$ and a permutation $\alpha_{j} \in \mathcal{F}_{\vec{\rho}_{j}}$ be given. A constrained local minimization, within the closed set $\Omega_{\alpha_{j}}$, is performed as follows. First, we determine a search direction

$$
\vec{p}_{j}=\underset{|p|=1}{\operatorname{argmin} \max }\left\{\left\langle p, \frac{\nabla A\left(\vec{\rho}_{j}\right)}{\left|\nabla A\left(\vec{\rho}_{j}\right)\right|}\right\rangle, \max _{s \in \mathcal{I}_{\vec{\rho}_{j}}^{\alpha_{j}}}\left\langle p, \frac{\nabla c_{s}^{\alpha_{j}}\left(\vec{\rho}_{j}\right)}{\left|\nabla c_{s}^{\alpha_{j}}\left(\vec{\rho}_{j}\right)\right|}\right\rangle\right\},
$$

where $\mathcal{I}_{\vec{\rho}_{j}}^{\alpha_{j}}$ is the index set of the constraints in (6.9)-(6.10) for which equality holds. We then consider two cases.

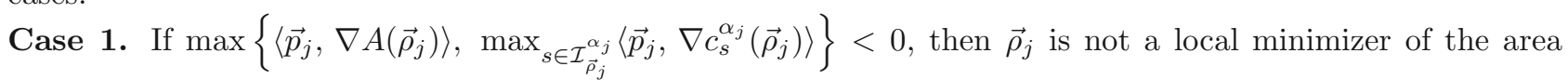
functional $A$ on $\Omega_{\alpha_{j}}$. In this case, we define the step length $\delta_{j}>0$ to be the largest value $\delta>0$ such that

$$
\begin{aligned}
A\left(\vec{\rho}_{j}+\delta \vec{p}_{j}\right) & \leq A\left(\vec{\rho}_{j}\right)+c \delta\left\langle\nabla A\left(\vec{\rho}_{j}\right), \vec{p}_{j}\right\rangle ; \\
\vec{\rho}_{j}+\delta \vec{p}_{j} & \in \Omega_{\alpha_{j}}
\end{aligned}
$$

where $c \in] 0,1\left[\right.$ is a fixed constant. We then update: $\vec{\rho}_{j+1}=\vec{\rho}_{j}+d_{j} \vec{p}_{j}$, and set $\alpha_{j+1}=\alpha_{j}$. Step 2 is then repeated. 


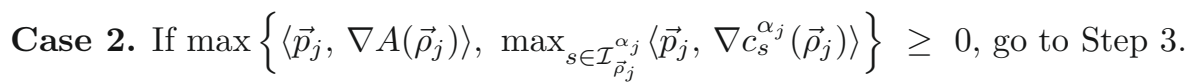

Step 3. If, for every permutation $\beta \in \mathcal{F}_{\vec{\rho}_{\alpha_{j}}}$, there holds

$$
\min _{|p|=1} \max \left\{\left\langle p, \frac{\nabla A\left(\vec{\rho}_{j}\right)}{\left|\nabla A\left(\vec{\rho}_{j}\right)\right|}\right\rangle, \max _{s \in \mathcal{I}_{\vec{\rho}_{j}}^{\beta}}\left\langle p, \frac{\nabla c_{s}^{\beta}\left(\vec{\rho}_{j}\right)}{\left|\nabla c_{s}^{\beta}\left(\vec{\rho}_{j}\right)\right|}\right\rangle\right\} \geq 0,
$$

go to Step 4.

Otherwise, we choose a permutation $\beta \in \mathcal{F}_{\vec{\rho}_{\alpha_{j}}}$ such that the above minimum is strictly negative and go to Step 2, replacing $\alpha_{j}$ with $\beta$.

Step 4. If the number of vertices $n_{j}$ of the polygonal is larger than a fixed integer $N$, we stop. Otherwise, we double the number of vertices and go back to Step 2. More precisely, if $\vec{\rho}_{j}=\left(\rho_{j, 1}, \ldots, \rho_{j, n_{j}}\right)$ determines the current polygon, we set $n_{j+1} \doteq 2 n_{j}$, and define $\vec{\rho}_{j+1} \doteq\left(\rho_{1}^{\prime}, \rho_{2}^{\prime}, \ldots, \rho_{2 n_{j}}^{\prime}\right)$. Here $\rho_{2 \ell}^{\prime}=\rho_{j, \ell}$, while $\rho_{2 \ell-1}^{\prime}$ is the radial coordinate (i.e. the distance to the origin) of the point $Q_{2 \ell-1}^{\prime}$ where the ray $\left\{\left(r \cos \frac{(2 \ell-1) \pi}{n_{j}}, r \sin \frac{(2 \ell-1) \pi}{n_{j}}\right) ; r \geq 0\right\}$ intersects the segment $S_{j, \ell}$ with endpoints

$$
Q_{\ell-1}=\rho_{j, \ell-1}\left(\cos \frac{2 \pi(\ell-1)}{n_{j}}, \sin \frac{2 \pi(\ell-1)}{n_{j}}\right), \quad Q_{\ell}=\rho_{j, \ell}\left(\cos \frac{2 \pi \ell}{n_{j}}, \sin \frac{2 \pi \ell}{n_{j}}\right) .
$$

Remark. The line search algorithm described in Step 2 is somewhat different from the ones more commonly used in the literature [12]. In our numerical experiments, it appears to perform better for the specific problem at hand.

\subsection{Numerical results}

\subsubsection{The isotropic case}

We first consider the case where the initial burned set $R_{0}$ is the open unit disc, and the fire propagates in all directions with unit speed. This yields a minimum time function $T(x)=|x|-1$. We assume that the wall can be constructed at speed $\sigma=4$, and seek a strategy that minimizes the total burned area (with no cost associated to the length of the walls).

We compute a numerical solution symmetric w.r.t. the $x_{1}$-axis, taking, as initial guess, a polygonal with 24 vertices located along the curve

$$
\gamma=\left\{2 e^{\theta / \sqrt{3}}(\cos \theta, \sin \theta) ; \theta \in[0, \pi]\right\} .
$$

The numerical results, using polygonals with up to $N=185$ vertices, are illustrated in Figure 13.

A symmetric analytical solution to this minimum-area problem was found in [3]. The optimal wall consists of an arc of circumference and two logarithmic spirals, enclosing a region (on the half plane) with area $A_{\min } \approx$ 15.4337. The solution computed by the algorithm has a similar shape. However, it encloses an area $A \approx 16.8103$. We expect that a better accuracy could be gained by further increasing the number of vertices of the polygon.

\subsubsection{The ice-cream cone case}

Next, consider a non-isotropic case. Here the initial set $R_{0}$ is again the open unit disc, while the set $F$ of fire propagation velocities is defined as in (6.6). In the numerical experiments we take $\kappa=3$, while the construction speed of the wall is $\sigma=4.1$. As shown in [6], for $x=\left(x_{1}, x_{2}\right) \in R_{\infty}$ the minimum time function is

$$
T(x)=\frac{\left(\kappa x_{1}+1\right)-\sqrt{\left(\kappa x_{1}+1\right)^{2}-\left(\kappa^{2}-1\right)\left(x_{1}^{2}+x_{2}^{2}-1\right)}}{\kappa^{2}-1} .
$$




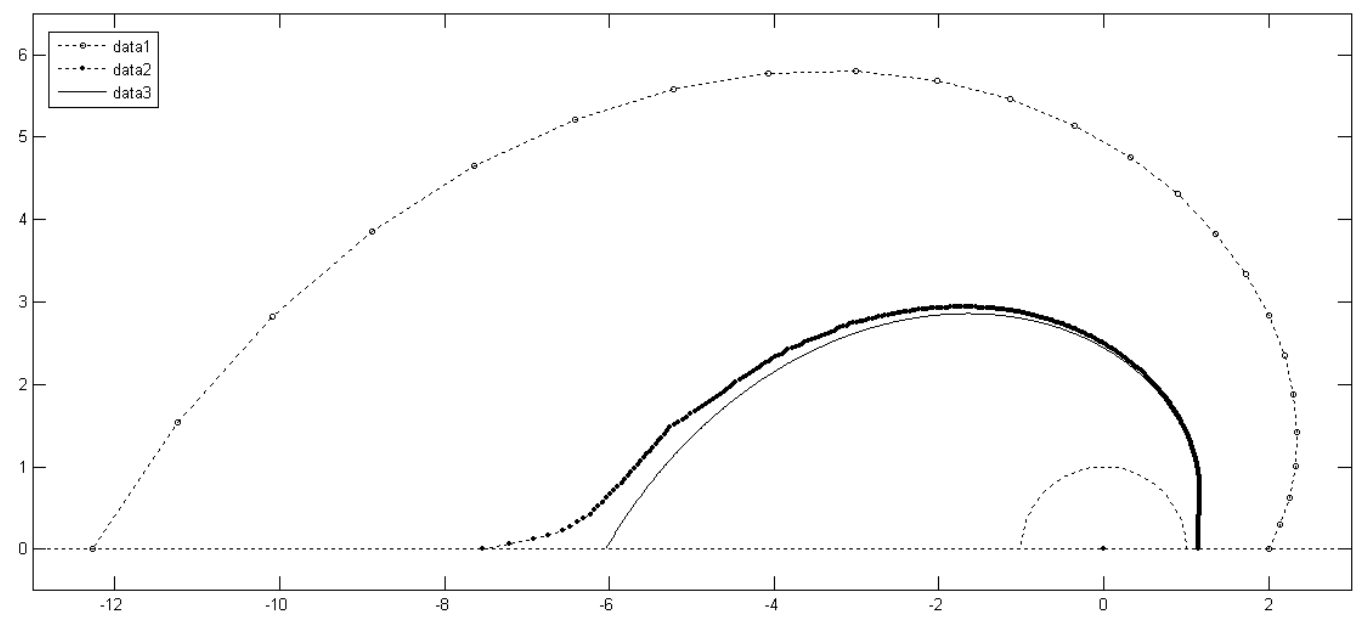

Figure 13. The isotropic case. Here the initial guess is a polygonal with 24 vertices, located along a logarithmic spiral. The thick dotted line gives the numerically computed solution. The thin solid line is the analytic solution found in [3].

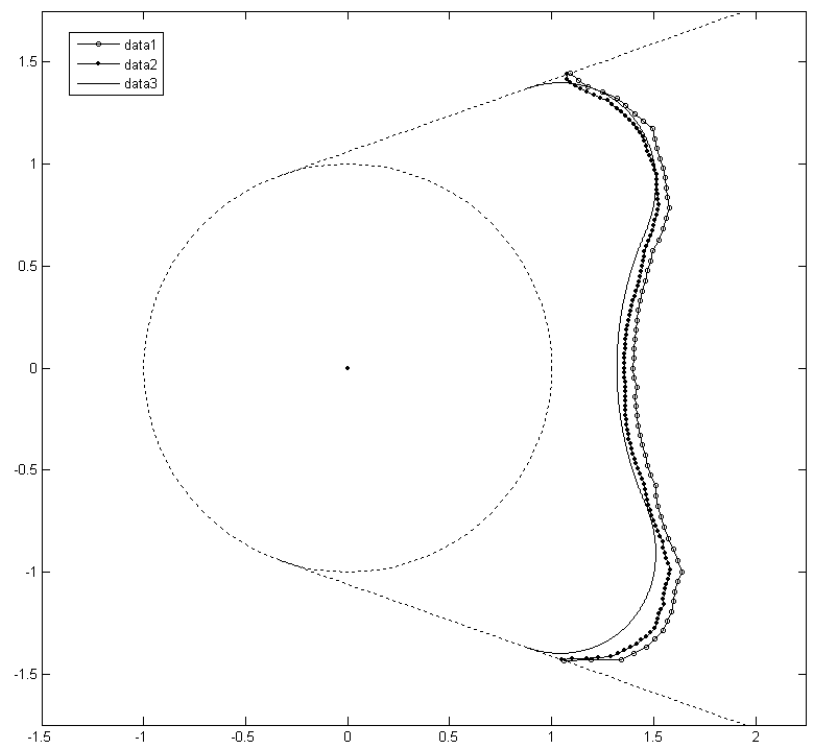

Figure 14. Minimizing the total burned area in the "ice-cream cone" case. The solid line shows the analytical solution. The two dotted lines represent two polygonal approximations generated by the algorithm, with $N=64$ and $N=128$ vertices, respectively.

In our first numerical experiment, we seek a strategy which minimizes the total burned area. For this problem, the theoretical analysis in [6] shows that the optimal shape of the wall, symmetric w.r.t. the $x_{1}$-axis, consists of an arc of circumference (constructed away from the fire) and of two additional arcs (constructed along the edge of the advancing fire front). This minimizer is plotted as the solid line (data3) in Figure 14. The area of burned region associated with this strategy is $A_{\min } \approx 2.0458$. The two dotted curves in Figure 14 show 


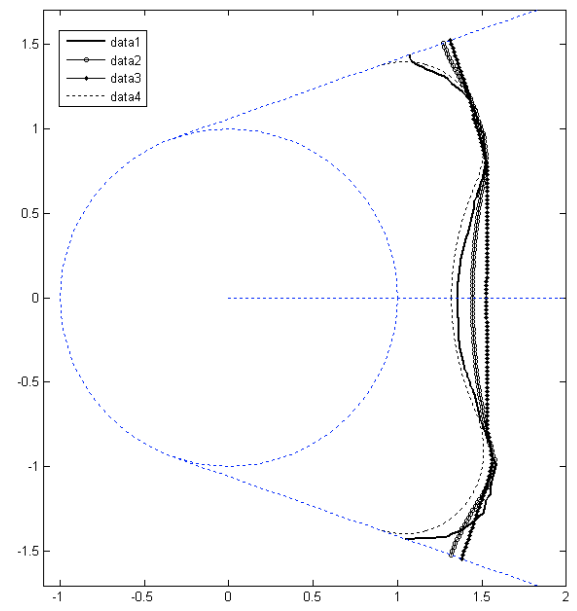

Figure 15. The "ice-cream cone" case with different cost functions. The dashed line (data4) shows the analytical solution with $\lambda=0$. Data 1,2, 3 provide the numerical solutions for $\lambda=0, \lambda=1$ and $\lambda=5$, respectively.

two approximate solutions obtained by the numerical algorithm, taking the vertical segment

$$
\mathcal{S} \doteq\left\{\left(x_{1}, x_{2}\right) ; \quad x_{1}=\frac{16 \sqrt{2}}{3}-3,\left|x_{2}\right| \leq \frac{8}{3}\right\}
$$

as initial guess. The first (data1 in the figure) is a polygonal curve with $N=64$ vertices, yielding a burned area $A_{64} \approx 2.3025$. The second (data2) is a polygonal with $N=128$ vertices, yielding a burned area $A_{128} \approx 2.1596$.

In the last set of numerical simulations, we consider different cost functionals, also penalizing the total length of the wall. Figure 15 shows numerical solutions to the optimization problem (6.4) with $\lambda=0,1,5$ respectively. As the parameter $\lambda$ increases, one can see an increase of the total burned area and a decrease of the total length of the wall.

Acknowledgements. This work was supported by NSF through grant DMS-0807420, "New problems in nonlinear control". The authors also wish to thank Camillo De Lellis for useful suggestions.

\section{REFERENCES}

[1] L. Ambrosio, N. Fusco and D. Pallara, Functions of Bounded Variation and Free Discontinuity Problems. Oxford University Press (2000).

[2] J.P. Aubin and A. Cellina, Differential Inclusions. Springer-Verlag, Berlin (1984).

[3] A. Bressan, Differential inclusions and the control of forest fires. J. Differ. Equ. 243 (2007) 179-207 (special volume in honor of A. Cellina and J. Yorke).

[4] A. Bressan and C. De Lellis, Existence of optimal strategies for a fire confinement problem. Comm. Pure Appl. Math. 62 (2009) 789-830.

[5] A. Bressan and T. Wang, The minimum speed for a blocking problem on the half plane. J. Math. Anal. Appl. 356 (2009) $133-144$

[6] A. Bressan, M. Burago, A. Friend and J. Jou, Blocking strategies for a fire control problem. Anal. Appl. 6 (2008) $229-246$.

[7] C. De Lellis, Rectifiable Sets, Densities and Tangent Measures, Zürich Lectures in Advanced Mathematics. EMS Publishing House (2008).

[8] H. Federer, Geometric Measure Theory. Springer-Verlag, New York (1969).

[9] M. Henle, A Combinatorial Introduction to Topology. W.H. Freeman, San Francisco (1979).

[10] K. Kuratovski. Topology, Vol. II. Academic Press, New York (1968).

[11] W.S. Massey, A Basic Course in Algebraic Topology. Springer-Verlag, New York (1991).

[12] J. Nocedal and S.J. Wright. Numerical Optimization. Springer, New York (2006). 